\title{
Arginine-rich C9ORF72 ALS proteins stall ribosomes in a manner distinct from a canonical ribosome-associated quality control substrate
}

\author{
Viacheslav Kriachkov ${ }^{1}$, Hamish E G McWilliam ${ }^{1,2}$, Justine D Mintern ${ }^{1}$, Shanika L Amarasinghe ${ }^{3}$, Matt \\ Ritchie $^{3}$, Luc Furic ${ }^{4,5,6}$, Danny M Hatters ${ }^{1, *}$ \\ ${ }^{1}$ Department of Biochemistry and Pharmacology and Bio21 Molecular Science and Biotechnology \\ Institute. The University of Melbourne, Victoria 3010 Australia \\ ${ }^{2}$ Department of Microbiology and Immunology, Peter Doherty Institute of Infection and Immunity, \\ The University of Melbourne, Parkville, Victoria, Australia.
${ }^{3}$ Walter and Eliza Hall Institute of Medical Research, Victoria 3052 Australia
${ }^{4}$ Translational Prostate Cancer Research Laboratory, Peter MacCallum Cancer Centre, Melbourne, Victoria, Australia, 3000 \\ ${ }^{5}$ Sir Peter MacCallum Department of Oncology, University of Melbourne, Parkville, Victoria, \\ Australia 3010 \\ ${ }^{6}$ Monash Biomedicine Discovery Institute Cancer Program, Department of Anatomy and \\ Developmental Biology, Monash University, Clayton, Victoria, Australia, 3800
}

*Correspondence: dhatters@unimelb.edu (D.M. Hatters).

\begin{abstract}
Hexanucleotide expansion mutations in C9ORF72 are a cause of familial amyotrophic lateral sclerosis. We previously reported that long arginine-rich dipeptide repeats (DPR), mimicking abnormal proteins expressed from the hexanucleotide expansion, caused translation stalling when expressed in cell culture models. Whether this stalling provides a mechanism of pathogenicity remains to be determined. Here we explored the molecular features of DPR-induced stalling and examined whether known regulatory mechanisms of ribosome quality control (RQC) are involved to sense and resolve the stalls. We demonstrate that arginine-containing DPRs lead to stalling in a length dependent manner, with lengths longer than 40 repeats invoking severe translation arrest. Mutational screening of $40 \times G l y-X x x$ DPRs shows that stalling is most pronounced where $X x x$ are positively charged amino acids (Arg or Lys). Through a genome-wide knockout screen we find that genes regulating stalling on polyadenosine mRNA coding for poly-Lys, a canonical RQC substrate, respond differently to the readthrough of arginine-rich DPRs. Indeed, we find evidence that DPR-mediated stalling has no natural regulatory responses even though the stalls may be sensed, as evidenced by an upregulation of RQC gene expression. These findings therefore implicate arginine-rich DPR-mediated stalled ribosomes as posing a particular danger to cellular health and viability.
\end{abstract}

\section{INTRODUCTION}

GGGGCC hexanucleotide repeat expansion mutations in intron 1 of C9ORF72 are the cause of familial amyotrophic lateral sclerosis (ALS) and frontotemporal dementia $(1,2)$. Normally, there are less than 24 repeats, whereas the length is expanded to often hundreds in ALS-causing alleles (3). The expanded GGGGCC leads to the production of sense and antisense mRNA products that display two unusual features that have been postulated to contribute to disease. One is that the mRNA can form granular intracellular foci that contribute to toxicity through RNA-based mechanisms (4). The other 
is that both sense and antisense mRNA can be translated through alternative initiation codons (i. e. non-AUG translation) to produce five distinct dipeptide-containing polymers (poly-GA, GR, GP, PR and PA) (5). These abnormal proteins accumulate in the brain of patients with C9ORF72 mutations. The two arginine containing dipeptide repeats (DPR), poly-GR and poly-PR, have been shown to be particularly toxic when added to cells or when expressed in cellular and organismal models (6-8). The toxicity is preserved if the GR and PR are encoded by mixed codons, suggesting that the protein sequence itself is directly toxic, and therefore not entirely arising from the mRNA $(7,8)$.

The fidelity of protein synthesis involves mechanisms that detect and eliminate spontaneous cases of aberrant translation (9). This includes: translation of mRNAs with defects including mRNAs with stable stem-loop structures, damaged bases or other obstacles to elongation (no-go decay, NGD); mRNAs that lack stop codons (nonstop decay, NSD) and mRNAs with premature stop codons (nonsense-mediated mRNA decay, NMD) (10). A marked decrease in elongation rates on such mRNAs can lead to formation of persistent ribosomal collisions, which are targeted for ribosome-associated quality control (RQC) pathway that resolves such stalls and causes degradation of both defective mRNA and polypeptide (11-14). In the event stalls happen aberrantly and remain unresolved they can have pathological consequences. It was reported that simultaneous mutation in CNS (central nervous system)-specific tRNA gene that leads to stalling on specific codons and loss of GTPBP2, a ribosome rescue factor resolving such stalls, causes widespread neurodegeneration in mice (15). Similarly, mice with a loss of function of LTN1 protein that targets stalled nascent chains for degradation also exhibited a neurodegenerative phenotype (16).

Recent studies from us and others showed that expression of mRNA encoding long repeats of polyGR and poly-PR causes severe ribosomal stalling $(17,18)$ and it was proposed that the positively charged R-rich nascent polypeptide electrostatically jams the ribosome exit tunnel (19). However, the mechanisms by which stalling occurs and whether it contributes to toxicity remain unclear. Another outstanding question is whether known mechanisms that target stalled ribosomes in cells are able to sense and modify stalls caused by poly-GR and poly-PR. Here we examine the molecular features of stalling from poly-PR. We examined the length of the repeat required to stall and specificity of arginine in a context of DPR-mediated arrest as well as conducted a screen for potential genetic regulators of stalling on poly-PR protein. Collectively our findings indicated that the mechanisms involved in stalling caused by long R-rich DPRs are unequivocally distinct to the mechanisms leading to stalling on poly- $K$ coded by polyadenosine sequence that mimics defective mRNAs lacking stop codons. Our findings point to a mechanism of toxicity that relates to a lack of an evolved capacity to resolve stalls involving R-rich DPRs.

\section{MATERIALS AND METHODS}

\section{DNA constructs}

Plasmids coding linker, poly-K, $102 \times G R$ and $102 \times P R$ in dual fluorescence stall reporter were prepared as described (17). cDNA for 20x, 30x, 40x, 50x, 75× GR and PR, and 40× GA, GC, GD, GE, GF, GH, GI, GK, GL, GM, GN, GP, GQ, GS, GT, GV, GW, GY were synthesized and cloned into dual fluorescence stall reporter (GenScript). For knockout cell pools, sgRNA sequences targeting genes of interest were designed using Benchling software. They were cloned into lentiCRISPRv2 vector (a gift from Feng Zhang; Addgene plasmid \# 52961) (20) using BsmBI restriction sites, as outlined in the protocol from Zhang lab (available at Addgene). For stable Tet-inducible cell lines, GFP-P2A-ChFP, GFP-P2A-poly-K and GFP-P2A-102×PR were cloned by restriction digestion and ligation and inserted into pLVXTetOne-Puro vector (Takara Bio \# 631849) using Gibson assembly. All cloned constructs were 
validated by sequencing. DNA preparations were made with Stbl3 E. coli cells (Thermo Fisher). The sequence information is available (Table S1).

\section{Cell Culture}

HEK293T cells, obtained originally from the American Type Culture Collection (ATCC, Manassas, Virginia), were maintained in complete DMEM (Dulbecco's modified Eagle medium (DMEM) supplemented with $10 \% \mathrm{v} / \mathrm{v}$ fetal calf serum and $1 \%$ GlutaMAX). Cells were cultured in a humidified incubator with $5 \% \mathrm{v} / \mathrm{v}$ atmospheric $\mathrm{CO}_{2}$ at $37^{\circ} \mathrm{C}$. For the drug treatment experiments, harringtonine (Abcam \# ab141941; 20 mM working stock in DMSO) or cycloheximide (Sigma-Aldrich \# 01810-1G; $50 \mathrm{mM}$ working stock in DMSO) were added to cells 6 hours post-transfection alongside a change of culture medium, and cells were analyzed by FACS at 24 hours post-transfection.

\section{Ribosomal stalling assays}

HEK293T cells were transfected with staller constructs using Lipofectamine 3000 reagent and harvested $24 \mathrm{~h}$ post-transfection. Cells were analyzed using LSRFortessa X-20 flow cytometer (BD Biosciences). Side and forward scatter height, width, and area were collected to gate for single live cell population. GFP fluorescence were collected with the 488-nm laser and FITC (530/30) filter to gate for transfected cells, ChFP fluorescence was collected with the 561-nm laser and mCherry (610/20) filter. Flow cytometric gating and data analysis was performed using FlowJo software (v10.5.3) and graphs were analyzed in RStudio (v. 1.4.1106) and GraphPad Prism 8. Median FITC-A and median mCherry-A fluorescence values were used to calculate ChFP/GFP ratio for each sample. All ratios were normalized to average of ChFP/GFP ratios for fluorescence reporter with linker sequence (negative no-stall control).

\section{Confocal imaging}

HEK293T cells were transfected with GFP-tagged poly-PR constructs using Lipofectamine 3000 reagent. 24 hours after transfection, cells were fixed in 4\% paraformaldehyde for 15 min at room temperature. Nuclei were counterstained with Hoechst 33342 at 1:750 dilution (Thermo Fisher \# H3570) for 20 min then washed twice in PBS. Confocal images were obtained on Zeiss Elyra LSM880 confocal microscope using $63 \times$ oil-immersion objective lens at room temperature. Single color controls were used to establish and adjust to remove bleed through of the emission filter bandwidths. FIJI version of ImageJ (v.1.53c) (21) was used for image processing.

\section{Lentiviral work}

Lentivirus for Brunello knock out library, single knock out cell lines and Tet-inducible cell lines were made per Thermo Fisher guidelines with minor modifications. HEK293T cells were transfected with pCMV-VSV-G (a gift from Bob Weinberg; Addgene plasmid \# 8454) (22), psPAX2 (a gift from Didier Trono; Addgene plasmid \# 12260) and lentiviral transfer plasmid in equimolar ratios using Lipofectamine 3000 reagent. Virus-containing media was collected 48 hours post-transfection and filtered using $0.45 \mu \mathrm{m}$ filter. Virus supernatant was stored at $-80^{\circ} \mathrm{C}$. HEK293T cells were transduced with virus in the presence of $8 \mu \mathrm{g} / \mathrm{mL}$ polybrene. The media was refreshed with complete DMEM 24 $\mathrm{h}$ after transduction. At $48 \mathrm{~h}$ after transduction, antibiotic selection for infected cells were performed with $1 \mu \mathrm{g} / \mathrm{ml}$ puromycin (Thermo Fisher \# A1113803; $10 \mathrm{mg} / \mathrm{mL}$ working stock in $20 \mathrm{mM}$ HEPES buffer) for 3 days.

\section{RNA sequencing}

HEK293K cell lines harbouring the constructs under the Tet-on expression system were induced for expression by adding complete DMEM media containing $500 \mathrm{ng} / \mu \mathrm{l}$ doxycycline (Sigma-Aldrich \# D9891; $1 \mathrm{mg} / \mathrm{ml}$ working stock in DMSO) to the cells for $48 \mathrm{~h}$ prior to harvesting. Expression was confirmed by microscopy and flow cytometry for the fluorescent proteins. For each construct, non- 
induced cells were collected in parallel to the doxycycline-induced cells. Cell were collected in icecold lysis buffer ( $20 \mathrm{mM}$ Tris pH 7.4, $150 \mathrm{mM} \mathrm{NaCl}, 5 \mathrm{mM} \mathrm{MgCl}, 1 \mathrm{mM} \mathrm{DTT}, 100$ mg/ml cycloheximide, $1 \% \mathrm{v} / \mathrm{v}$ Triton X-100 and $25 \mathrm{U} / \mathrm{ml}$ Turbo DNase I), triturated ten times through a 26-gauge needle and centrifuged for $10 \mathrm{~min}$ at $20,000 \mathrm{~g}$ at $4{ }^{\circ} \mathrm{C}$. The RNA concentration in the supernatant was measured using Promega QuantiFluor RNA System. Total RNA was isolated by Zymo Research Direct-zol RNA MiniPrep Kit. The stranded mRNA library was prepared and sequenced on Illumina Novaseq 6000 $100 \mathrm{bp}$ SR by the Australian Genome Research Facility. Triplicate samples were prepared. Count tables were generated in Galaxy.org using HISAT2 (v.2.1.0) algorithm (23), and the list of differentially expressed genes were generated with DESeq2 package (v.1.30.1) (24) in R (v.4.0.5). Gene set enrichment analysis (GSEA) was performed using a javaGSEA desktop application (v.4.1.0) $(25,26)$. Curated gene set GSEA: C5.GO.BP.v7.4 was obtained from the Molecular Signatures Database (MSigDB) (27). Data sets with FDR $<0.1$ were considered as statistically significant. Enrichment map visualization was performed in Cytoscape (v.3.8.0) (28).

\section{Genome-wide CRISPR knockout screening}

The human CRISPR knockout pooled library Brunello was obtained from Addgene (a gift from David Root and John Doench; Addgene \# 73178) (29). The experiment was done as outlined in the following protocol (30), with minor modifications. For each of 3 replicates, HEK293T cells were transduced at $\mathrm{MOI}=0.4$. Following antibiotic selection, at least $10^{8}$ cells were transfected with either poly-K or $102 \times P R$ staller. 24 hours post-transfection half of cells were subjected to sorting while the other half was left as unsorted control population. Cells were sorted at BD InFlux cell sorter for top $5 \%$ of high and low ChFP/GFP ratio populations. gDNA from collected cells was extracted with salt precipitation protocol described here (31). The sgRNA-containing cassettes were amplified as described previously (32). The pooled amplicons were sequenced using an Illumina NovaSeq 6000. The results were analyzed and false discovery rate (FDR) and log2 fold change (LFC) values for each gene were calculated using MAGeCK (v.0.5.9.2) computational tool (33). Functional enrichment analysis was done in Cytoscape (v.3.8.0) for all screen hits with FDR<0.05.

\section{Generation of knockout cell pools}

Single sgRNA expression vectors were cloned and lentivirus production with the following transduction was performed as described above. More than $50 \%$ knockout efficiency for each pool was validated by ICE-seq analysis (34) after isolating total gDNA and sequencing the targeted region of gene of interest. The sgRNA sequences targeting each selected gene used are shown in Table S1. Cells expressing a non-targeting sgRNA were used as a control pool. Fold changes in ChFP/GFP ratio for poly-K, 102×PR or $102 \times$ GR staller after individual gene knockout were calculated by dividing the ratio from cells expressing sgRNA to ratio from cells without sgRNA. For each knockout pool, fold changes in ChFP/GFP on a staller sequence were compared against fold changes in ChFP/GFP on a linker sequence that does not cause stalling.

\section{Statistical analysis}

Details of quantification including number of samples analyzed and statistical tests used are described in the corresponding figure legends and methods section.

\section{RESULTS}

\section{Arginine-rich dipeptide repeats stall during translation in a length-dependent manner}

To find the dipeptide repeat length at which the nascent chain is long enough to hinder ribosome readthrough, we used a series of DNA sequences coding PR and GR dipeptide repeats of various 
lengths: 10x, 20x, 30x, 40x, 50x, 75x, and 102x. All DPR-coding sequences were codon-optimized to minimize repetitive mRNA sequences and codon repetitiveness. Readthrough efficiency was assessed using the previously described dual-reporter transcript (Figure 1A) (35-38). Briefly, this reporter consists of mRNA sequence to be tested for stalling which is flanked, in frame and without stop codons, with sequences encoding green fluorescent protein (GFP) and mCherry fluorescent protein (ChFP). P2A sequences separate the test sequence from the GFP and ChFP and cause the ribosome to "skip" the glycyl-prolyl peptide bond formation. As such, a single mRNA transcript can translate three separate proteins (39). The yield of ChFP versus GFP allows a measure of whether the test sequence can modulate stalling, i.e. the ChFP/GFP ratio will be reduced in the event stalling occurs. While both poly-PR and poly-GR proteins lowered the ChFP/GFP ratio progressively in a lengthdependent manner suggesting increased levels of stalled translation, they also showed different trends, with poly-GR appeared to stall more substantially at the longer lengths of dipeptide repeat (Figure 1B, C).

Poly-PR is known for its property to form nuclear foci (7) and to test if this phenotype changes with

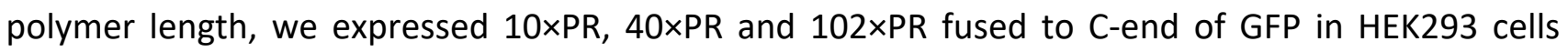

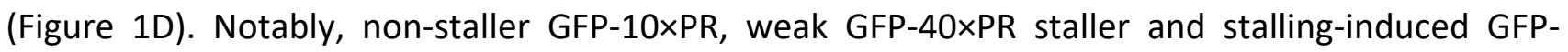
$102 \times P R$ had similar localization patterns. Overall, this finding suggests that poly-PR confers a functional property for recruitment to bodies in the nucleus, however, this feature appears independent to stalling or to the repeat length.

\section{Specificity of arginine in the context of DPR-mediated ribosome stalling}

Prior work has suggested that positively charged amino acid patterning can influence the stalling of translation during synthesis (40). Given that other non-arginine DPRs associated with C9ORF72 hexanucleotide expansion (poly-PA and poly-GA) do not appear to lead to stalling (17), we next examined if stalling in a DPR context was specific to arginine. A small library of DPRs was assessed in the stalling assay whereby each variant was a DPR containing one of the other 19 canonical amino acids alternating with glycine. The sequences were codon optimized to minimize secondary structures and codon usage bias where possible. The DPRs containing charged residues at neutral pH (Arg, Asp, Glu and Lys) led to the largest decreases in ChFP/GFP ratios, suggestive of substantial ribosome stalling (Figure 2A-B, Figure S1). Of these, the negatively charged DPRs (40×GD and 40×GE)

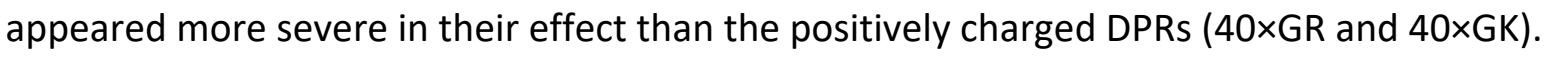

It was previously found that the translation of open reading frames containing multiple acidic residues at the start of the sequence results in intrinsic ribosomal destabilization, which leads to translation abortion in E. coli (41). Such a mechanism, which is distinct to stalling, may lead to selective reduction of ChFP in our assay and therefore confound interpretation of stalling. To examine for this possibility, we inserted a linker sequence at the $\mathrm{N}$-terminus of the DPR sequences

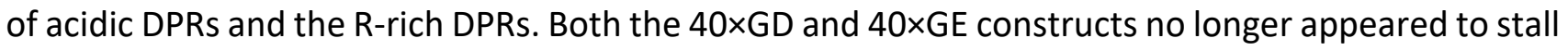

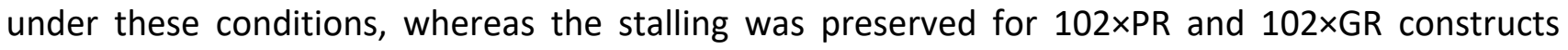
(Figure $2 \mathrm{C}$ ). We therefore concluded that stalling in the context of DPR is selective to positively charged (arginine and lysine) moieties.

\section{poly-PR expression leads to significant changes in the expression of various stress response genes including the upregulation of $R Q C$ genes at transcriptional level}

To determine whether polyadenosine-mediated stalling induces similar change in gene expression as poly-PR stalling, we performed transcriptome analysis. We created three stable HEK293T cell lines expressing GFP-P2A-ChFP (which provides a negative control with no stalling), GFP-P2A-poly-K, 
whereby poly- $K$ was coded with (AAA) codons (and therefore represents the canonical RQC substrate, which induces stalling), and GFP-P2A-102×PR under a Tet-inducible promoter (Figure 3A). Gene expression levels were compared for the poly-K or 102×PR staller versus the ChFP negative control. Principle component analysis indicated that 102xPR-expressing cells clustered separately to all the other sample groups as well as the cells that were uninduced (Figure 3B). Volcano plots revealed that expression of polyadenosine led to relatively minor changes in gene expression, whereas $102 \times P R$ expression led to substantial changes (Figure 3C). Indeed, 102×PR expression resulted in 9962 genes significantly changing expression compared to cells expressing ChFP. Of these, 5086 genes were significantly upregulated and of these 1037 more than 2-fold. 4876 genes were significantly downregulated and of these 647 genes more than 2 -fold (Table S2). For the poly-K staller, 303 genes were significantly upregulated, but none more than 2 -fold. 102 genes were down-regulated, and of these only 2 showed more than 2 -fold decrease.

Gene set enrichment analysis identified 170 Gene Ontology (GO) terms that were significantly upregulated in the 102×PR-expressing cells and $327 \mathrm{GO}$ terms that were downregulated (Table S2). Further pathway enrichment analysis (Figure S2) revealed that many GO terms related to ribosome biogenesis, RNA metabolism, RNA processing, RNA transport and RNA modification (mostly methylation) were upregulated in response to 102×PR expression (Figure 3D). It has been reported that the expression of poly-PR inhibits global translation possibly due to interactions of poly-PR with ribosomes and other components of the translation machinery (42-44). As such, our results may reflect a compensatory response to activate genes involved in global translation. Other upregulated GO terms were linked to cytoskeleton which is in the agreement with prior findings of poly-PR disturbing the organization of the cytoskeleton (45) and interacting with actin-related cytoskeletal proteins (17). Our data also supports the role for poly-PR in inhibiting DNA double strand break repair pathways (46) as we saw an upregulation of GO terms related to mitotic cell cycle such as negative regulation of nuclear division (GO:0051784), negative regulation of metaphase/anaphase transition of cell cycle (GO:1902100), negative regulation of chromosome organization (GO:2001251) and mitotic intra-S DNA damage checkpoint signaling (GO:0031573).

In addition, many metabolic pathways were downregulated as a result of 102×PR expression. Prominent were clusters of GO terms related to glycoprotein metabolism, lipid biosynthesis and other terms related to cellular metabolic processes. These findings suggest substantial metabolic adaptation to the $102 \times \mathrm{PR}$ expression. This result is consistent with previous reports finding that polyPR directs promiscuous proteome binding and cytotoxocity $(17,43)$. The other point of note was the significant upregulation of RQC-associated genes as well as some genes involved in integrated stress response (ISR) (Figure 3E). These effects were not seen with the poly-K construct. This finding suggested that stalling of poly-K coded by polyadenosine can be readily accommodated by proteins that already present in cell whereas 102xPR stalled complexes are more detrimental to cell health and require a massive stress response from a cell.

\section{Genome-wide knockout screen unmasks a distinct signature of regulators of $102 \times P R$ readthrough compared to poly-K readthrough}

To investigate whether mechanisms that are known to regulate stalls on polyadenosine mRNA are involved in sensing and responding to poly-PR stalls, we performed a genome-wide CRISPR/Cas9 knockout screen using the Brunello library, which targets 19,114 human genes at a coverage of 4 gRNA targets per gene (29). We performed two screens: one with the 102×PR staller reporter and the other with poly-K coded by (AAA) codons as a reference for known RQC regulatory mechanisms $(37,47,48)$. Flow cytometry was used to sort and collect two groups of cells reflecting the higher and 
lower $5 \%$ ranges of ChFP/GFP ratios (Figure 4A). Population " $\mathrm{H}$ " represented cells with the highest ChFP/GFP ratios, which should encompass the highest readthrough levels. Population " $L$ " represented cells with lowest ChFP/GFP ratio, which should encompass the highest levels of stalling. The logic of the screen was that a knock-out of a particular gene involved in the resolution of stalling or RQC function would increase stalling (i.e. be over-represented in the L population). Conversely, gene knockouts of genes involved in pausing translation on stalled sequences, or recruitment of machinery to stalls, would be expected to be enriched in the $\mathrm{H}$ population, which depicts higher levels of readthrough.

The poly-K screen yielded 96 gene knockouts enriched in population $\mathrm{H}$ (of these 19 were enriched more than 2-fold), and 56 gene knockouts enriched in population $L$ (and of these 26 were more than 2-fold) (Figure 4B, Table S3). The 102×PR screen yielded 34 gene knockouts enriched in population $\mathrm{H}$ (and of these 5 were more than 2-fold) and 43 gene knockouts enriched in population $L$ (and of these 15 were more than 2 -fold). 21 gene knockouts were observed in common for both screens that increased apparent read-through $(\mathrm{H})$ and there were 12 genes in common that appeared to increase stalling (L). Figure $4 \mathrm{C}$ shows a further refined list of top gene knockouts for each staller which consists of gene knockouts that were significantly enriched in one population (FDR $<0.05, L F C>0)$ and simultaneously depleted from the opposite population (FDR $<0.05, \mathrm{LFC}<0)$.

The genes found in the poly-K screen included ZNF598, a previously known activator of the RQC (36), and members of ASC-1 complex (ASCC3, ASCC2 and TRIP4) that disassembles collided ribosomes that result from stalling on polyadenosine mRNA (49) (Table 1). These knockouts were enriched in population $\mathrm{H}$, which is consistent with other studies showing their knockout to improve poly-K readthrough $(36,37,49)$. Another gene involved in RQC activation, RACK1 (37), also appeared in our screen. RACK1 knockouts were significantly depleted from population $\mathrm{L}$ and on the threshold for significant enrichment in population $\mathrm{H}$ (a false discovery rate value of 0.059). Collectively, these findings provided confidence in the screen for finding the relevant knockouts that regulate mechanisms involved in sensing and resolving stalled ribosomes. It was previously shown that EIF4E2GIGYF2 (also known as 4EHP-GIGYF2) complex represses translation on defective mRNAs (50) and is recruited to ribosome collisions along with ZNF598 (51). According to our screen, cells with EIF4E2 and GIGYF2 knockouts appeared to have more stalling with the poly-K staller. This finding supports the hypothesis that in the absence of EIF4E2-GIGYF2 complex ZNF598 function on polyadenosine mRNA is enhanced.

Genes known to be involved in activating the RQC showed a distinct enrichment pattern in the $102 \times P R$ screen (Table 1). Notably, ZNF598 and RACK1 knockouts were enriched in population L which is the opposite to what we observed for poly-K staller. ASCC3, ASCC2, TRIP4 knockouts showed no enrichment in the $102 \times P R$ screen despite being hits in the poly-K screen. Other ribosome rescue factors seen in the polyK screen that can dissociate elongation complexes stalled at the 3 ' end of mRNA (HBS1L, PELOTA, ABCE1) (52), interact with ribosome collisions (EIF4E2, GIGYF2) or rescue ribosomes stalled at AGA arginine codons (GTPBP1, GTPBP2) (53) were not enriched in the $102 \times P R$ screen. These results suggested that co-translation and ribosome-associated quality control mechanisms that recognize and eliminate arrested ribosomes on polyadenosine sequences or other defective mRNAs might be unable to perform this function for poly-PR-induced stalls.

For further clues to pathways associated with genes identified in our screen we performed functional enrichment analysis. For both stallers, GO terms related to translation were enriched among gene knockouts improving the readthrough (Figure 5A-B). These are consistent with the key role of global translation since interference with translation strongly impacted the stall reporter behavior in our screen. This finding is supported by prior work in yeast showing that deletion of ribosomal proteins 
increases readthrough of poly-K and no-go decay (NGD) reporters (13). Knockouts of elongator complex (ELP3, ELP4, ELP5) and thiourydilase CTU1, which are required to efficiently decode AAA repeats by modifying U34 of lysyl-tRNA(UUU) (54), decreased the readthrough of poly-K. In addition, deletion of lysyl-tRNA synthetase (KARS) which catalyzes the formation of Lys-tRNA ${ }^{\text {Lys }}$ selectively increased stalling on poly-K, but not on $102 \times P R$ staller, which lacks Lys codons. The identification of these genes and pathways provide evidence the screen can identify proteins involved in both improving and reducing readthrough. Of note, genes activating RQC were identified in both screens, yet the genes showed different enrichment patterns further supporting our conclusions thus far that the surveillance and quality control mechanisms of stalling is dissimilar between polyadenosine and mRNA encoding 102×PR. Lastly, the enrichment of nuclear transport and nuclear envelope organization genes in $102 \times P R$ screen may relate to the recruitment of the poly-PR to the nucleus.

Next, we further examined the role of 14 genes identified in our screen by creating individual knockout cell pools (Figure 6, Table 2). These genes were chosen on the basis of having large log2 fold change values in the knockout screen (DRG2 and SKIV2L2 for poly-K; NPM1 and RPS25 for 102×PR; ABCF2, BOP1, DDX27, EIF2AK2, EIF6, NOP2 and RPL19 for both stallers), or having opposing effects between polyK and poly-PR (ZNF598, RACK1 and BANF1). To understand off-target impacts on the stall reporter readout, we included a control "linker" construct that contained a sequence known to not induce stalling (35-38). ZNF598 and RACK1 have been previously shown to increase readthrough of poly- $K$ coded by (AAA) codons when their levels are reduced $(36,37)$. Accordingly, their knockout increased the ChFP to GFP ratio by 3.7- and 2.7-fold, respectively, for the poly-K staller. By contrast, their knockout decreased the ratio for the 102×PR staller, in agreement with the screen outcomes which indicated that stalling was more pronounced when these genes are depleted. All other genes knockouts that impacted ChFP/GFP ratio on poly-K staller mostly had negligible or small effects on the corresponding ratios for the $102 \times P R$ readthrough. Collectively, these results indicated that the surveillance mechanisms involved in sensing stalls on polyadenosine mRNA have minimal effectivity in managing the stalls conferred by the 102×PR construct even if they may be able to sense the stalls as problematic based on their upregulation in the RNA-seq data.

To examine whether these effects of gene targeting were specific to arginine in the DPR, we tested the $102 \times G R$, which also causes stalling (17). None of the gene knockouts had any major effects on the ChFP/GFP ratio, suggesting that the stalling behaves similarly to that of $102 \times P R$ (Figure S3). However, the two knockouts that worsened stalling for 102×PR (RACK1 or ABCF2) appeared to have no effect on $102 \times G R$. This may be explained by the finding that $102 \times G R$ is more substantially stalled than $102 \times P R$ and hence has no capability to be further stalled (17) (Figure 1B, C).

\section{Inhibiting translation increases the readthrough of poly-K, but has limited or negative effects on R-rich DPRs translation}

While the effect of the 14 gene knockouts suggested that the canonical regulatory mechanisms overseeing stalls on polyadenosine mRNA do not seem to effectively resolve the R-rich DPR stalls, we did note that EIF6 and RPL19 knockouts yielded slightly increased ChFP/GFP ratios for both poly-K and poly-PR relative to the linker control (Figure 6). elF6 is a translation initiation factor that binds 605 ribosomal subunits which prevents them premature joining with 40 S subunits. Reduction of elF6 levels causes a decrease in the amount of available 60S subunits and impairs protein synthesis (55). We therefore hypothesized that elF6 knockout reduces ribosome load on mRNA which, according to the collision-based model, should mitigate arrest on stall sequences.

To test whether reducing the ribosome load and translational throughput on mRNA can allow underlying attenuated mechanisms capacity to correct the stalling phenotype, we performed a study 
modeled on prior experiments showing that reducing the number of ribosomal collisions increases protein output from some stall sequences and stabilizes their translation (13). First, we examined low concentrations of translation initiation inhibitor harringtonine to reduce the density of ribosomes on mRNAs (56). Harringtonine treatment led to an increase in ChFP/GFP ratios for poly-K, suggesting it increases its readthrough by reducing the occurrences of ribosome collisions (Figure 7A). This treatment also increased ChFP/GFP ratios for $102 \times \mathrm{PR}$, albeit to a lower extent, suggesting that while slowing ribosome density can be helpful to reduce $102 \times \mathrm{PR}$ stalling, mechanisms to address the stalls appear attenuated if they are indeed present. To explore this phenomenon with different approaches, we partly inhibited translation elongation with cycloheximide which appeared to improve the readthrough of the poly-K, however it led to a reduced apparent readthrough for the $102 \times P R$ (Figure 7B). Investigation of these treatments with other R-rich DPRs showed that the effects of reducing stalling were restricted to the longest repeat lengths we tested $(102 \times)$ and was more effective for PR compared to GR (Figure S4).

\section{DISCUSSION}

Collectively, the findings presented here support a mechanism by which R-rich DPRs lead to stalling and are unable to be resolved by established quality control mechanisms that address polyadenosine-mediated stalling. This is in agreement with previous studies showing that RQC does not regulate the expression of endogenous proteins with polybasic sequences (57). The implications of these findings point to R-rich DPR-mediated stalling having no natural regulatory response, and therefore the stalled ribosomes may pose a particular danger to cellular homeostasis and viability. Notably, stalling on long poly-PR proteins is exacerbated when the global translation in compromised by adding elongation inhibitor. A recent study found that ageing, which is accompanied by a decline in cellular proteostasis, causes greater ribosome pausing at polybasic stretches (58). This suggests that a limited capacity that cells have to cope with stalls induced by a long poly-PR protein may be worse upon ageing and/or in the presence of other stresses, and therefore could prove as critical in the biological pathways leading to disease onset and development.

A question that remains unsolved is why the stalls occur in the first place. One possibility is that emergent nascent chains of the R-rich DPRs can interfere in trans with other ribosomes in addition to directly blocking the ribosome during synthesis. Poly-K can efficiently stall when coded with 21 consecutive AAA codons, which would render the sequence occupying maximally about two thirds of the ribosome exit tunnel (36). The ribosome exit tunnel can hold about 30 amino acids of a linear peptide chain and up to 60 amino acids for a peptide in an $\alpha$-helical conformation (59). R-rich DPRs require more than 10 repeats (i.e. 20 amino acids for the poly-GR) and up to 40 repeats ( 80 amino acids for the poly-PR) to cause small levels of stalling (i.e. $25 \%$ or less). Such lengths would be sufficient to fill the ribosome exit tunnel and stall through an electrostatic jamming mechanism as previously proposed (17-19). However, the most effective stalling required longer dipeptide lengths ( $>30$ repeats for poly-GR ( 60 amino acids) and $>50$ repeats for poly-PR ( 100 amino acids)) to stall more than $50 \%$, and indeed did so in a length-dependent manner, suggesting that emergent DPR chain is involved in stalling. A preprint study suggested that the addition of short synthetic 20xPR and 20xGR polypeptides strongly inhibited global translation in vitro and occluded the exit tunnel of eukaryotic $80 \mathrm{~S}$ ribosomes (19). Accordingly, it is reasonable to predict that during R-rich DPR translation emergent poly-GR or poly-PR nascent chains could bind and block the exit tunnels of trailing ribosomes on the mRNA leading to obstruction of translation in trans, and a collateral aggregation of translational machinery. The support to this mechanism comes from poly-GR and poly-PR interactomes being enriched with translational proteins $(17,43)$. A further twist to this 
mechanism comes from simulations suggesting that the R-rich DPRs can phase separate once they reach a polymer length of about 25 repeats (60). Hence translation may be inhibited through a coalescence of ribosomes and blockage of their exit tunnels, which would presumably confer a great deal of toxic stress to cellular functioning.

Another aspect to our data is why the poly-GR appears to be more potent at stalling than poly-PR. One possibility is that the glycine allows greater conformational flexibility than proline (60). One consequence is that it may allow compact structures to accumulate in the ribosome exit tunnel, which may exacerbate blockage through electrostatic interactions. Indeed, the exit tunnel is wide enough to allow small protein domains to fold before exiting the ribosome tunnel (61). A greater flexibility may also lend to greater potency in influencing ribosomes in trans.

Poly-PR expression invokes a profound response from cells compared to poly-K expression, as reflected by substantial changes in mRNA levels for thousands of genes including genes involved in translation and cytoskeleton. Some of these responses likely arise from the multi-pronged toxicity previously implicated from poly-PR, including inhibition of translation (42-44) and the disruption of cytoskeleton architecture $(17,45,62)$. Other responses relate more directly to stress response pathways including ribosome quality control (RQC), integrated stress response (ISR), unfolded protein response (UPR) and heat shock response activator HSF1. The activation of RQC pathway is important since it suggests that mechanisms exist to recognize the stalls, yet our other findings suggest that they are unable to be adequately resolved. Indeed other work has pointed to a role for RQC protein ZNF598 promoting the cleavage of poly-GR (but not poly-PR) by the ubiquitinproteasome system while not affecting the readthrough efficiency (18). As such, the stalling may simply be overwhelming or improperly sensed by machinery that has not evolved to deal with such unnatural stalling sequences. In turn, it is plausible that the other stress responses are invoked in response to that inability to resolve through the RQC. Of note are studies finding DPR deposits in brain tissue to correlate with markers of the UPR (63).

\section{DATA AVAILABILITY}

The data from RNA sequencing and genome-wide knockout screening is available in Tables S2 and S3 respectively. RNA sequencing data from this study have been deposited to NCBI GEO with the accession code: GSE193962. All data supporting the findings of this study is available from the corresponding author upon request.

\section{CONFLICT OF INTEREST STATEMENT}

None declared.

\section{ACKNOWLEDGEMENTS}

This work was funded by grants to DMH (National Health and Medical Research Council APP1161803 and Australian Research Council DP170103093) and to LF (Department of Health and Human Services acting through the Victorian Cancer Agency (fellowship MCRF16007)). 


\section{REFERENCES}

1. DeJesus-Hernandez, M., Mackenzie, I.R., Boeve, B.F., Boxer, A.L., Baker, M., Rutherford, N.J., Nicholson, A.M., Finch, N.A., Flynn, H., Adamson, J. et al. (2011) Expanded GGGGCC hexanucleotide repeat in noncoding region of C9ORF72 causes chromosome 9p-linked FTD and ALS. Neuron, 72, 245256.

2. Renton, A.E., Majounie, E., Waite, A., Simon-Sanchez, J., Rollinson, S., Gibbs, J.R., Schymick, J.C., Laaksovirta, H., van Swieten, J.C., Myllykangas, L. et al. (2011) A hexanucleotide repeat expansion in C9ORF72 is the cause of chromosome 9p21-linked ALS-FTD. Neuron, 72, 257-268.

3. Van Mossevelde, S., van der Zee, J., Cruts, M. and Van Broeckhoven, C. (2017) Relationship between C9orf72 repeat size and clinical phenotype. Curr Opin Genet Dev, 44, 117-124.

4. McEachin, Z.T., Parameswaran, J., Raj, N., Bassell, G.J. and Jiang, J. (2020) RNA-mediated toxicity in C9orf72 ALS and FTD. Neurobiol Dis, 145, 105055.

5. Zu, T., Liu, Y., Banez-Coronel, M., Reid, T., Pletnikova, O., Lewis, J., Miller, T.M., Harms, M.B., Falchook, A.E., Subramony, S.H. et al. (2013) RAN proteins and RNA foci from antisense transcripts in C9ORF72 ALS and frontotemporal dementia. Proc Natl Acad Sci U S A, 110, E4968-4977.

6. Kwon, I., Xiang, S., Kato, M., Wu, L., Theodoropoulos, P., Wang, T., Kim, J., Yun, J., Xie, Y. and McKnight, S.L. (2014) Poly-dipeptides encoded by the C9orf72 repeats bind nucleoli, impede RNA biogenesis, and kill cells. Science, 345, 1139-1145.

7. Wen, X., Tan, W., Westergard, T., Krishnamurthy, K., Markandaiah, S.S., Shi, Y., Lin, S., Shneider, N.A., Monaghan, J., Pandey, U.B. et al. (2014) Antisense proline-arginine RAN dipeptides linked to C9ORF72ALS/FTD form toxic nuclear aggregates that initiate in vitro and in vivo neuronal death. Neuron, 84, 1213-1225.

8. Mizielinska, S., Gronke, S., Niccoli, T., Ridler, C.E., Clayton, E.L., Devoy, A., Moens, T., Norona, F.E., Woollacott, I.O.C., Pietrzyk, J. et al. (2014) C9orf72 repeat expansions cause neurodegeneration in Drosophila through arginine-rich proteins. Science, 345, 1192-1194.

9. Brandman, O. and Hegde, R.S. (2016) Ribosome-associated protein quality control. Nat Struct Mol Biol, 23, 7-15.

10. Joazeiro, C.A.P. (2017) Ribosomal Stalling During Translation: Providing Substrates for RibosomeAssociated Protein Quality Control. Annu Rev Cell Dev Biol, 33, 343-368.

11. Collart, M.A. and Weiss, B. (2020) Ribosome pausing, a dangerous necessity for co-translational events. Nucleic Acids Res, 48, 1043-1055.

12. Ikeuchi, K., Izawa, T. and Inada, T. (2018) Recent Progress on the Molecular Mechanism of Quality Controls Induced by Ribosome Stalling. Front Genet, 9, 743.

13. Simms, C.L., Yan, L.L. and Zaher, H.S. (2017) Ribosome Collision Is Critical for Quality Control during No-Go Decay. Mol Cell, 68, 361-373 e365.

14. Sitron, C.S. and Brandman, O. (2020) Detection and Degradation of Stalled Nascent Chains via Ribosome-Associated Quality Control. Annu Rev Biochem, 89, 417-442.

15. Ishimura, R., Nagy, G., Dotu, I., Zhou, H., Yang, X.L., Schimmel, P., Senju, S., Nishimura, Y., Chuang, J.H. and Ackerman, S.L. (2014) RNA function. Ribosome stalling induced by mutation of a CNS-specific tRNA causes neurodegeneration. Science, 345, 455-459.

16. Bengtson, M.H. and Joazeiro, C.A. (2010) Role of a ribosome-associated E3 ubiquitin ligase in protein quality control. Nature, 467, 470-473.

17. Radwan, M., Ang, C.S., Ormsby, A.R., Cox, D., Daly, J.C., Reid, G.E. and Hatters, D.M. (2020) Arginine in C9ORF72 Dipolypeptides Mediates Promiscuous Proteome Binding and Multiple Modes of Toxicity. Mol Cell Proteomics, 19, 640-654.

18. Park, J., Lee, J., Kim, J.H., Lee, J., Park, H. and Lim, C. (2021) ZNF598 co-translationally titrates poly(GR) protein implicated in the pathogenesis of C9ORF72-associated ALS/FTD. Nucleic Acids Res.

19. Loveland, A.B., Svidritskiy, E., Susorov, D., Lee, S., Park, A., Demo, G., Gao, F.-B. and Korostelev, A.A. (2020) Ribosome inhibition by C9ORF72-ALS/FTD-associated poly-PR and poly-GR proteins revealed by cryo-EM. bioRxiv. 
20. Sanjana, N.E., Shalem, O. and Zhang, F. (2014) Improved vectors and genome-wide libraries for CRISPR screening. Nat Methods, 11, 783-784.

21. Schindelin, J., Arganda-Carreras, I., Frise, E., Kaynig, V., Longair, M., Pietzsch, T., Preibisch, S., Rueden, C., Saalfeld, S., Schmid, B. et al. (2012) Fiji: an open-source platform for biological-image analysis. Nat Methods, 9, 676-682.

22. Stewart, S.A., Dykxhoorn, D.M., Palliser, D., Mizuno, H., Yu, E.Y., An, D.S., Sabatini, D.M., Chen, I.S., Hahn, W.C., Sharp, P.A. et al. (2003) Lentivirus-delivered stable gene silencing by RNAi in primary cells. $R N A$, 9, 493-501.

23. Kim, D., Langmead, B. and Salzberg, S.L. (2015) HISAT: a fast spliced aligner with low memory requirements. Nat Methods, 12, 357-360.

24. Love, M.I., Huber, W. and Anders, S. (2014) Moderated estimation of fold change and dispersion for RNA-seq data with DESeq2. Genome Biol, 15, 550.

25. Subramanian, A., Tamayo, P., Mootha, V.K., Mukherjee, S., Ebert, B.L., Gillette, M.A., Paulovich, A., Pomeroy, S.L., Golub, T.R., Lander, E.S. et al. (2005) Gene set enrichment analysis: a knowledge-based approach for interpreting genome-wide expression profiles. Proc Natl Acad Sci U S A, 102, 1554515550.

26. Mootha, V.K., Lindgren, C.M., Eriksson, K.F., Subramanian, A., Sihag, S., Lehar, J., Puigserver, P., Carlsson, E., Ridderstrale, M., Laurila, E. et al. (2003) PGC-1alpha-responsive genes involved in oxidative phosphorylation are coordinately downregulated in human diabetes. Nat Genet, 34, 267273.

27. Liberzon, A., Birger, C., Thorvaldsdottir, H., Ghandi, M., Mesirov, J.P. and Tamayo, P. (2015) The Molecular Signatures Database (MSigDB) hallmark gene set collection. Cell Syst, 1, 417-425.

28. Shannon, P., Markiel, A., Ozier, O., Baliga, N.S., Wang, J.T., Ramage, D., Amin, N., Schwikowski, B. and Ideker, T. (2003) Cytoscape: a software environment for integrated models of biomolecular interaction networks. Genome Res, 13, 2498-2504.

29. Doench, J.G., Fusi, N., Sullender, M., Hegde, M., Vaimberg, E.W., Donovan, K.F., Smith, I., Tothova, Z., Wilen, C., Orchard, R. et al. (2016) Optimized sgRNA design to maximize activity and minimize offtarget effects of CRISPR-Cas9. Nat Biotechnol, 34, 184-191.

30. Joung, J., Konermann, S., Gootenberg, J.S., Abudayyeh, O.O., Platt, R.J., Brigham, M.D., Sanjana, N.E. and Zhang, F. (2017) Genome-scale CRISPR-Cas9 knockout and transcriptional activation screening. Nat Protoc, 12, 828-863.

31. Chen, S., Sanjana, N.E., Zheng, K., Shalem, O., Lee, K., Shi, X., Scott, D.A., Song, J., Pan, J.Q., Weissleder, R. et al. (2015) Genome-wide CRISPR screen in a mouse model of tumor growth and metastasis. Cell, $160,1246-1260$.

32. Sanson, K.R., Hanna, R.E., Hegde, M., Donovan, K.F., Strand, C., Sullender, M.E., Vaimberg, E.W., Goodale, A., Root, D.E., Piccioni, F. et al. (2018) Optimized libraries for CRISPR-Cas9 genetic screens with multiple modalities. Nat Commun, 9, 5416.

33. Li, W., Xu, H., Xiao, T., Cong, L., Love, M.I., Zhang, F., Irizarry, R.A., Liu, J.S., Brown, M. and Liu, X.S. (2014) MAGeCK enables robust identification of essential genes from genome-scale CRISPR/Cas9 knockout screens. Genome Biol, 15, 554.

34. Hsiau, T., Maures, T., Waite, K., Yang, J., Kelso, R., Holden, K. and Stoner, R. (2018) Inference of CRISPR Edits from Sanger Trace Data. bioRxiv.

35. Brandman, O., Stewart-Ornstein, J., Wong, D., Larson, A., Williams, C.C., Li, J.W., Zhou, S., King, D., Shen, P.S., Weibezahn, J. et al. (2012) A Ribosome-Bound Quality Control Complex Triggers Degradation of Nascent Peptides and Signals Translation Stress. Cell, 151, 1042-1054.

36. Juszkiewicz, S. and Hegde, R.S. (2017) Initiation of Quality Control during Poly(A) Translation Requires Site-Specific Ribosome Ubiquitination. Mol Cell, 65, 743-750 e744.

37. Sundaramoorthy, E., Leonard, M., Mak, R., Liao, J., Fulzele, A. and Bennett, E.J. (2017) ZNF598 and RACK1 Regulate Mammalian Ribosome-Associated Quality Control Function by Mediating Regulatory 40S Ribosomal Ubiquitylation. Mol Cell, 65, 751-760 e754. 
38. Garzia, A., Jafarnejad, S.M., Meyer, C., Chapat, C., Gogakos, T., Morozov, P., Amiri, M., Shapiro, M., Molina, H., Tuschl, T. et al. (2017) The E3 ubiquitin ligase and RNA-binding protein ZNF598 orchestrates ribosome quality control of premature polyadenylated mRNAs. Nat Commun, 8, 16056.

39. Lin, Y.J., Huang, L.H. and Huang, C.T. (2013) Enhancement of heterologous gene expression in Flammulina velutipes using polycistronic vectors containing a viral $2 \mathrm{~A}$ cleavage sequence. PLoS One, 8, e59099.

40. Lu, J. and Deutsch, C. (2008) Electrostatics in the ribosomal tunnel modulate chain elongation rates. J Mol Biol, 384, 73-86.

41. Chadani, Y., Niwa, T., Izumi, T., Sugata, N., Nagao, A., Suzuki, T., Chiba, S., Ito, K. and Taguchi, H. (2017) Intrinsic Ribosome Destabilization Underlies Translation and Provides an Organism with a Strategy of Environmental Sensing. Mol Cell, 68, 528-539 e525.

42. Lee, K.H., Zhang, P., Kim, H.J., Mitrea, D.M., Sarkar, M., Freibaum, B.D., Cika, J., Coughlin, M., Messing, J., Molliex, A. et al. (2016) C9orf72 Dipeptide Repeats Impair the Assembly, Dynamics, and Function of Membrane-Less Organelles. Cell, 167, 774-788 e717.

43. Hartmann, H., Hornburg, D., Czuppa, M., Bader, J., Michaelsen, M., Farny, D., Arzberger, T., Mann, M., Meissner, F. and Edbauer, D. (2018) Proteomics and C9orf72 neuropathology identify ribosomes as poly-GR/PR interactors driving toxicity. Life Sci Alliance, 1, e201800070.

44. Moens, T.G., Niccoli, T., Wilson, K.M., Atilano, M.L., Birsa, N., Gittings, L.M., Holbling, B.V., Dyson, M.C., Thoeng, A., Neeves, J. et al. (2019) C9orf72 arginine-rich dipeptide proteins interact with ribosomal proteins in vivo to induce a toxic translational arrest that is rescued by elF1A. Acta Neuropathol, 137, 487-500.

45. Shiota, T., Nagata, R., Kikuchi, S., Nanaura, H., Matsubayashi, M., Nakanishi, M., Kobashigawa, S., Nagayama, K., Sugie, K., Yamashiro, Y. et al. (2020) C9orf72- derived proline:arginine poly-dipeptides disturb cytoskeletal architecture. bioRxiv.

46. Andrade, N.S., Ramic, M., Esanov, R., Liu, W., Rybin, M.J., Gaidosh, G., Abdallah, A., Del'Olio, S., Huff, T.C., Chee, N.T. et al. (2020) Dipeptide repeat proteins inhibit homology-directed DNA double strand break repair in C9ORF72 ALS/FTD. Mol Neurodegener, 15, 13.

47. Chandrasekaran, V., Juszkiewicz, S., Choi, J., Puglisi, J.D., Brown, A., Shao, S., Ramakrishnan, V. and Hegde, R.S. (2019) Mechanism of ribosome stalling during translation of a poly(A) tail. Nat Struct Mol Biol, 26, 1132-1140.

48. Tesina, P., Lessen, L.N., Buschauer, R., Cheng, J., Wu, C.C., Berninghausen, O., Buskirk, A.R., Becker, T., Beckmann, R. and Green, R. (2020) Molecular mechanism of translational stalling by inhibitory codon combinations and poly(A) tracts. EMBO J, 39, e103365.

49. Juszkiewicz, S., Speldewinde, S.H., Wan, L., Svejstrup, J.Q. and Hegde, R.S. (2020) The ASC-1 Complex Disassembles Collided Ribosomes. Mol Cell, 79, 603-614 e608.

50. Hickey, K.L., Dickson, K., Cogan, J.Z., Replogle, J.M., Schoof, M., D'Orazio, K.N., Sinha, N.K., Hussmann, J.A., Jost, M., Frost, A. et al. (2020) GIGYF2 and 4EHP Inhibit Translation Initiation of Defective Messenger RNAs to Assist Ribosome-Associated Quality Control. Mol Cell, 79, 950-962 e956.

51. Juszkiewicz, S., Slodkowicz, G., Lin, Z., Freire-Pritchett, P., Peak-Chew, S.Y. and Hegde, R.S. (2020) Ribosome collisions trigger cis-acting feedback inhibition of translation initiation. Elife, $\mathbf{9}$.

52. Pisareva, V.P., Skabkin, M.A., Hellen, C.U., Pestova, T.V. and Pisarev, A.V. (2011) Dissociation by Pelota, $\mathrm{Hbs} 1$ and $\mathrm{ABCE} 1$ of mammalian vacant $80 \mathrm{~S}$ ribosomes and stalled elongation complexes. EMBO J, 30, 1804-1817.

53. Terrey, M., Adamson, S.I., Gibson, A.L., Deng, T., Ishimura, R., Chuang, J.H. and Ackerman, S.L. (2020) GTPBP1 resolves paused ribosomes to maintain neuronal homeostasis. Elife, $\mathbf{9}$.

54. Rapino, F., Zhou, Z., Roncero Sanchez, A.M., Joiret, M., Seca, C., El Hachem, N., Valenti, G., Latini, S., Shostak, K., Geris, L. et al. (2021) Wobble tRNA modification and hydrophilic amino acid patterns dictate protein fate. Nat Commun, 12, 2170.

55. Pesce, E., Miluzio, A., Turcano, L., Minici, C., Cirino, D., Calamita, P., Manfrini, N., Oliveto, S., Ricciardi, S., Grifantini, R. et al. (2020) Discovery and Preliminary Characterization of Translational Modulators that Impair the Binding of elF6 to 60S Ribosomal Subunits. Cells, 9. 
56. Ingolia, N.T., Lareau, L.F. and Weissman, J.S. (2011) Ribosome profiling of mouse embryonic stem cells reveals the complexity and dynamics of mammalian proteomes. Cell, 147, 789-802.

57. Barros, G.C., Requiao, R.D., Carneiro, R.L., Masuda, C.A., Moreira, M.H., Rossetto, S., Domitrovic, T. and Palhano, F.L. (2021) Rqc1 and other yeast proteins containing highly positively charged sequences are not targets of the RQC complex. J Biol Chem, 296, 100586.

58. Stein, K.C., Morales-Polanco, F., van der Lienden, J., Rainbolt, T.K. and Frydman, J. (2022) Ageing exacerbates ribosome pausing to disrupt cotranslational proteostasis. Nature.

59. Kramer, G., Boehringer, D., Ban, N. and Bukau, B. (2009) The ribosome as a platform for cotranslational processing, folding and targeting of newly synthesized proteins. Nature Structural and Molecular Biology, 16, 589+.

60. Jafarinia, H., van der Giessen, E. and Onck, P.R. (2020) Phase Separation of Toxic Dipeptide Repeat Proteins Related to C9orf72 ALS/FTD. Biophys J, 119, 843-851.

61. Nilsson, O.B., Hedman, R., Marino, J., Wickles, S., Bischoff, L., Johansson, M., Muller-Lucks, A., Trovato, F., Puglisi, J.D., O'Brien, E.P. et al. (2015) Cotranslational Protein Folding inside the Ribosome Exit Tunnel. Cell Rep, 12, 1533-1540.

62. Fumagalli, L., Young, F.L., Boeynaems, S., De Decker, M., Mehta, A.R., Swijsen, A., Fazal, R., Guo, W., Moisse, M., Beckers, J. et al. (2021) C9orf72-derived arginine-containing dipeptide repeats associate with axonal transport machinery and impede microtubule-based motility. Sci Adv, 7, eabg3013.

63. Gami-Patel, P., van Dijken, I., Meeter, L.H., Melhem, S., Morrema, T.H.J., Scheper, W., van Swieten, J.C., Rozemuller, A.J.M., Dijkstra, A.A. and Hoozemans, J.J.M. (2021) Unfolded protein response activation in C9orf72 frontotemporal dementia is associated with dipeptide pathology and granulovacuolar degeneration in granule cells. Brain Pathol, 31, 163-173.

64. Juszkiewicz, S., Chandrasekaran, V., Lin, Z., Kraatz, S., Ramakrishnan, V. and Hegde, R.S. (2018) ZNF598 Is a Quality Control Sensor of Collided Ribosomes. Mol Cell, 72, 469-481 e467.

65. Hashimoto, S., Sugiyama, T., Yamazaki, R., Nobuta, R. and Inada, T. (2020) Identification of a novel trigger complex that facilitates ribosome-associated quality control in mammalian cells. Sci Rep, 10, 3422.

66. Ishimura, R., Nagy, G., Dotu, I., Zhou, H., Yang, X.L., Schimmel, P., Senju, S., Nishimura, Y., Chuang, J.H. and Ackerman, S.L. (2014) Ribosome stalling induced by mutation of a CNS-specific tRNA causes neurodegeneration. Science, 345, 455-459.

67. Segura-Totten, M., Kowalski, A.K., Craigie, R. and Wilson, K.L. (2002) Barrier-to-autointegration factor: major roles in chromatin decondensation and nuclear assembly. $J$ Cell Biol, 158, 475-485.

68. Rohrmoser, M., Holzel, M., Grimm, T., Malamoussi, A., Harasim, T., Orban, M., Pfisterer, I., GruberEber, A., Kremmer, E. and Eick, D. (2007) Interdependence of Pes1, Bop1, and WDR12 controls nucleolar localization and assembly of the PeBoW complex required for maturation of the $60 \mathrm{~S}$ ribosomal subunit. Mol Cell Biol, 27, 3682-3694.

69. Kellner, M., Rohrmoser, M., Forne, I., Voss, K., Burger, K., Muhl, B., Gruber-Eber, A., Kremmer, E., Imhof, A. and Eick, D. (2015) DEAD-box helicase DDX27 regulates 3' end formation of ribosomal 47S RNA and stably associates with the PeBoW-complex. Exp Cell Res, 334, 146-159.

70. Jang, S.H., Kim, A.R., Park, N.H., Park, J.W. and Han, I.S. (2016) DRG2 Regulates G2/M Progression via the Cyclin B1-Cdk1 Complex. Mol Cells, 39, 699-704.

71. Pakos-Zebrucka, K., Koryga, I., Mnich, K., Ljujic, M., Samali, A. and Gorman, A.M. (2016) The integrated stress response. EMBO Rep, 17, 1374-1395.

72. Ceci, M., Gaviraghi, C., Gorrini, C., Sala, L.A., Offenhauser, N., Marchisio, P.C. and Biffo, S. (2003) Release of elF6 (p27BBP) from the 60S subunit allows 80 S ribosome assembly. Nature, 426, 579-584.

73. Wang, H., Wang, L., Wang, Z., Dang, Y., Shi, Y., Zhao, P. and Zhang, K. (2020) The nucleolar protein NOP2 is required for nucleolar maturation and ribosome biogenesis during preimplantation development in mammals. FASEB J, 34, 2715-2729.

74. Box, J.K., Paquet, N., Adams, M.N., Boucher, D., Bolderson, E., O'Byrne, K.J. and Richard, D.J. (2016) Nucleophosmin: from structure and function to disease development. BMC Mol Biol, 17, 19. 
bioRxiv preprint doi: https://doi.org/10.1101/2022.02.09.479805; this version posted February $10,2022$. The copyright holder for this

preprint (which was not certified by peer review) is the author/funder, who has granted bioRxiv a license to display the preprint in perpetuity. It is made available under aCC-BY-NC-ND 4.0 International license.

75. Yamada, S.B., Gendron, T.F., Niccoli, T., Genuth, N.R., Grosely, R., Shi, Y., Glaria, I., Kramer, N.J., Nakayama, L., Fang, S. et al. (2019) RPS25 is required for efficient RAN translation of C9orf72 and other neurodegenerative disease-associated nucleotide repeats. Nat Neurosci, 22, 1383-1388.

76. Meola, N., Domanski, M., Karadoulama, E., Chen, Y., Gentil, C., Pultz, D., Vitting-Seerup, K., LykkeAndersen, S., Andersen, J.S., Sandelin, A. et al. (2016) Identification of a Nuclear Exosome Decay Pathway for Processed Transcripts. Mol Cell, 64, 520-533. 


\section{TABLE AND FIGURE LEGENDS}

\section{Table 1. Enrichment factors for genes involved in rescue of stalled ribosomes.}

\begin{tabular}{|c|c|c|c|c|c|c|c|c|c|c|c|}
\hline \multirow{3}{*}{$\begin{array}{c}\text { Gene } \\
\text { name }\end{array}$} & \multirow{3}{*}{ Gene function } & \multicolumn{5}{|c|}{ poly-K } & \multicolumn{5}{|c|}{ 102xPR } \\
\hline & & \multicolumn{2}{|c|}{$\begin{array}{l}\text { H: high } \\
\text { ChFP/GFP }\end{array}$} & \multicolumn{2}{|c|}{$\begin{array}{l}\text { L: low } \\
\text { ChFP/GFP }\end{array}$} & \multirow{2}{*}{$\begin{array}{c}\text { effect } \\
\text { of } \\
\text { gene } \\
\text { KO }\end{array}$} & \multicolumn{2}{|c|}{$\begin{array}{c}\text { H: high } \\
\text { ChFP/GFP }\end{array}$} & \multicolumn{2}{|c|}{$\begin{array}{c}\text { L: low } \\
\text { ChFP/GFP }\end{array}$} & \multirow{2}{*}{$\begin{array}{c}\text { effect } \\
\text { of } \\
\text { gene } \\
\text { KO }\end{array}$} \\
\hline & & FDR $^{*}$ & LFC\# & FDR & LFC & & FDR & LFC & FDR & LFC & \\
\hline ZNF598 & $\begin{array}{r}\text { ubiquitin-ligase, recog } \\
\text { them for AS }\end{array}$ & 2E-04 & 0.98 & 8E-04 & $\mid-1.35$ & read & 0.69 & -0.28 & $2 \mathrm{E}-04$ & 0.76 & stall \\
\hline RACK1 & $\begin{array}{r}\begin{array}{r}\text { component of the } 40 \mathrm{~S} s \\
\text { on collii }\end{array} \\
\end{array}$ & 0.059 & 0.56 & 8E-04 & $\mid-1.79$ & read & 0.08 & $-0.65 \mid$ & $2 \mathrm{E}-04$ & 1.73 & stall \\
\hline ASCC3 & $\begin{array}{r}\text { helicase, a member } \\
\text { collid }\end{array}$ & $2 \mathrm{E}-04$ & 1.27 & $3 \mathrm{E}-03$ & $\mid-1.58$ & read & 1 & -0.13 & 0.43 & 0.55 & - \\
\hline ASCC2 & $\begin{array}{r}\text { ubiquitin-binding } \\
\text { disass }\end{array}$ & $2 \mathrm{E}-04$ & 1.02 & 0.48 & $\mid-0.46$ & read & 1 & 0.11 & 0.99 & 0.22 & - \\
\hline TRIP4 & $\begin{array}{r}\text { a member of ASC-1 complex } \\
\text { ribosomes, suggested to } \\
\text { complex to rit }\end{array}$ & 1E-03 & 0.59 & $2 \mathrm{E}-03$ & $\mid-1.26$ & read & 0.95 & -0.19 & 0.17 & 0.40 & - \\
\hline EIF4E2 & \multirow{2}{*}{$\begin{array}{l}\text { EIF4E2-GIGYF2 (also known as 4EHP-GIGYF2) complex } \\
\text { represses translation on defective mRNAs (46) and is } \\
\text { recruited to ribosome collisions along with ZNF598 (47) }\end{array}$} & 0.92 & 0.33 & $1 \mathrm{E}-03$ & 1.05 & stall & 0.99 & -0.05 & 0.75 & 0.33 & - \\
\hline GIGYF2 & & 0.02 & -0.72 & 2E-04 & 1.36 & stall & 0.81 & -0.10 & 0.84 & 0.31 & - \\
\hline HBS1L & \multirow{3}{*}{$\begin{array}{l}\text { HBS1L-PELO complex in the presence of ABCE1 } \\
\text { dissociates ribosomes stalled at the end of a truncated } \\
\text { mRNA and therefore lacking mRNA in the A-site }(49,52)\end{array}$} & 1 & $\mid-0.07$ & 0.13 & -0.41 & - & 1 & 0.19 & 0.79 & $\mid-0.31$ & - \\
\hline PELO & & 0.69 & 0.28 & 0.06 & -0.87 & - & 0.98 & 0.24 & 0.87 & $\mid-0.34$ & - \\
\hline ABCE1 & & 1 & 0.22 & 1 & -0.11 & - & 1 & -0.07 & 0.79 & 0.44 & - \\
\hline GTPBP1 & \multirow{2}{*}{$\begin{array}{l}\text { resolve codon-specific ribosome pausing on AGA codons } \\
(53,66)\end{array}$} & 1 & 0.16 & 0.93 & -0.24 & - & 1 & 0.07 & 1 & -0.05 & - \\
\hline STPBP2 & & 0.74 & 0.13 & 0.70 & -0.23 & - & 0.95 & \begin{tabular}{|l|}
0.29 \\
\end{tabular} & 1 & \begin{tabular}{|l|}
-0.09 \\
\end{tabular} & - \\
\hline
\end{tabular}

"False discovery rate; "log2 fold change; effect of gene KO (on stalling): read = KO facilitates readthrough on a staller sequence, stall $=\mathrm{KO}$ leads to more stalling on a staller sequence 
bioRxiv preprint doi: https://doi.org/10.1101/2022.02.09.479805; this version posted February 10,2022 . The copyright holder for this

preprint (which was not certified by peer review) is the author/funder, who has granted bioRxiv a license to display the preprint in perpetuity. It is made available under aCC-BY-NC-ND 4.0 International license.

Table 2. Validation of screen hits by individual knockouts

\begin{tabular}{|c|c|c|c|c|c|c|c|}
\hline \multirow{3}{*}{ gene } & \multirow{3}{*}{ function } & \multicolumn{6}{|c|}{$\mathrm{KO}$ effect on staller readthrough } \\
\hline & & \multicolumn{2}{|c|}{ in screen ${ }^{1}$} & \multicolumn{2}{|c|}{$\begin{array}{l}\text { individual KO cell } \\
\text { pool, compared to } \\
\text { control cells }{ }^{2}\end{array}$} & \multicolumn{2}{|c|}{$\begin{array}{c}\text { individual KO cell } \\
\text { pool, compared to } \\
\text { linker }{ }^{3}\end{array}$} \\
\hline & & poly-K & 102xPR & poly-K & 102xPR & poly-K & 102xPR \\
\hline ABCF2 & unknown, a member of $A B C$ transporters family & stall & stall & stall *** & stall $* * *$ & stall *** & stall *** \\
\hline BANF1 & nuclear assembly and chromatin organization (67) & stall & read & stall *** & n.s. & stall *** & read *** \\
\hline BOP1 & $60 S$ ribosome subunit biogenesis (68) & read & read & read *** & read *** & read $^{* * *}$ & n.s. \\
\hline $\mathrm{DD} \times 27$ & RNA helicase involved in rRNA processing (69) & read & read & read $* * *$ & read $* * *$ & read $^{* * *}$ & n.s. \\
\hline DRG2 & unknown, important role in cell growth (70) & stall & n.s. & stall *** & stall *** & stall *** & stall *** \\
\hline EIF2AK2 & $\begin{array}{c}\text { (also: PKR) inhibits cellular translation during } \\
\text { stress }(71)\end{array}$ & stall & stall & stall *** & n.s. & stall *** & n.s. \\
\hline EIF6 & $\begin{array}{l}\text { ribosome biogenesis, binds to } 60 S \text { to prevent } \\
\text { premature association with } 40 S \text { subunit }(72)\end{array}$ & read & read & read $^{* * *}$ & read $^{* * *}$ & read $^{* * *}$ & read ** \\
\hline NOP2 & $\begin{array}{l}\text { nucleolar maturation and ribosome biogenesis } \\
(73)\end{array}$ & read & read & read *** & read $^{* * *}$ & read $^{* * *}$ & n.s. \\
\hline NPM1 & RNA transport and ribosome biogenesis $(74)$ & n.s. & stall & read $^{* * *}$ & n.s. & read *** & stall *** \\
\hline RACK1 & $\begin{array}{l}\text { scaffolding protein, component of } 40 \text { S ribosome; } \\
\text { activator of RQC on polyadenosine mRNA (37) }\end{array}$ & n.s. & stall & read $^{* * *}$ & stall *** & read $^{* * *}$ & stall *** \\
\hline RPL19 & $60 S$ ribosomal protein & read & read & read $* * *$ & read *** & read $* * *$ & read *** \\
\hline RPS25 & $\begin{array}{c}\text { 40S ribosomal protein, required for efficient RAN } \\
\text { translation of C9orf72 repeats }(75)\end{array}$ & n.s. & stall & stall *** & stall *** & stall *** & stall *** \\
\hline SKIV2L2 & $\begin{array}{c}\text { (also: MTREX) RNA helicase, associated with the } \\
\text { RNA exosome complex (76) }\end{array}$ & read & n.s. & read *** & n.s. & read $^{* * *}$ & stall *** \\
\hline ZNF598 & activator of RQC on polyadenosine mRNA (36) & read & stall & read $^{* * *}$ & stall *** & read $^{* * *}$ & stall *** \\
\hline
\end{tabular}

${ }^{1}$ based on the screen results, read = significantly enriched in $\mathrm{H}$ population, stall = significantly enriched in $\mathrm{L}$ population.

${ }^{2,3}$ comparisons were made between log2fold changes (LFC) in ChFP/GFP. For each knockout, LFC $=\log 2$ (the ratio in cells with sgRNA targeting an indicated gene divided by the ratio in non-infected cells without sgRNA). n.s. - not significant; ${ }^{* *} \mathrm{P}<0.01{ }^{* * *} \mathrm{P}<0.0001$, as determined by one-way ANOVA and Dunnett's post hoc test $(n=4)$. 2 based on the comparison between LFC for KO cell pool and control cell pool (cells with non-targeting sgRNA). ${ }^{3}$ based on the comparison between LFC for KO cell pool expressing an indicated staller and LFC for the same KO cell pool expressing linker sequence that does not cause stalling. 


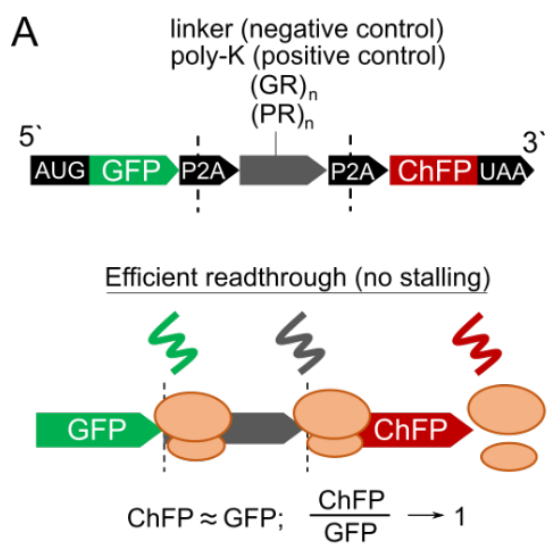

Impaired readthrough (ribosome stalling)

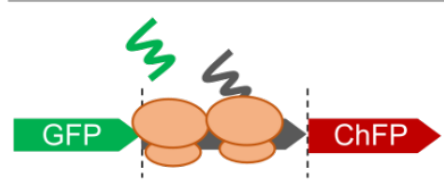

ChFP $<$ GFP; $\quad \frac{\text { ChFP }}{\text { GFP }} \rightarrow 0$
B
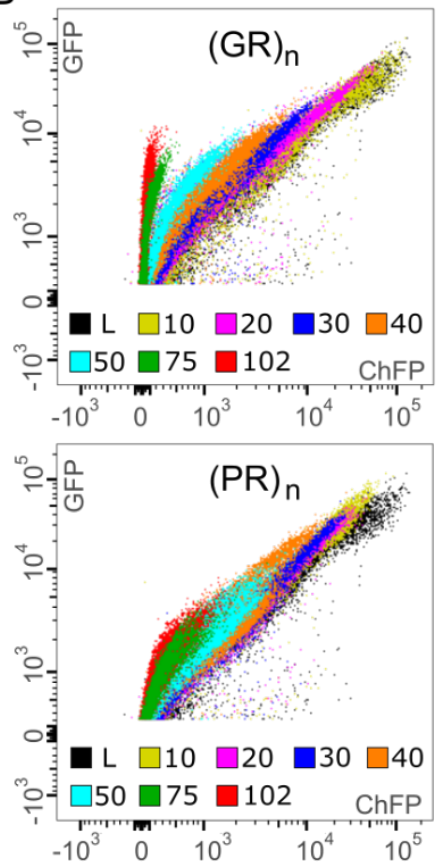

Merged
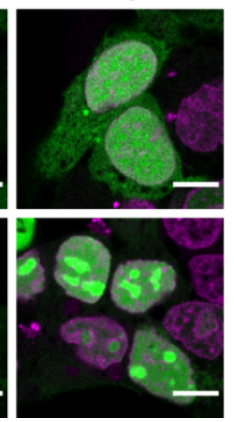

C
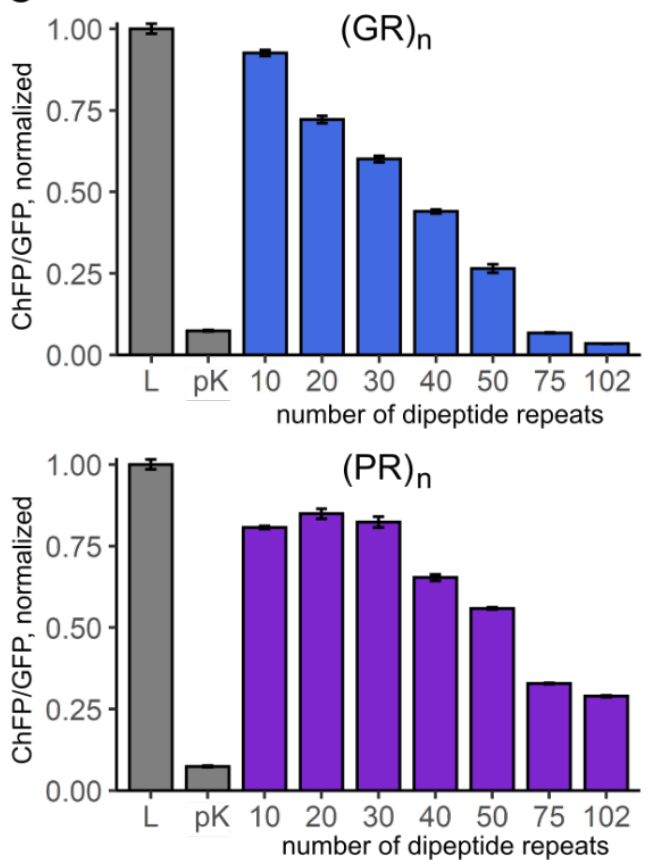

Nucleus

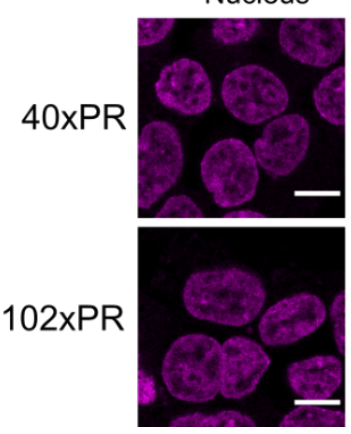

GFP-PR
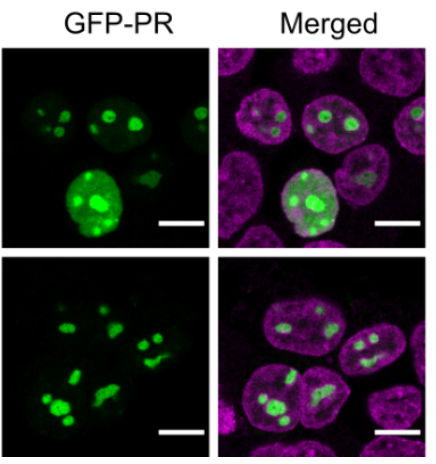

Figure 1. Ribosome stalling assays with poly-GR and poly-PR of different lengths. (A) A schematic of the sequence of the dual-fluorescent stall reporter. (B) Flow cytograms of HEK293T cells after $24 \mathrm{~h}$ of transfection with poly-GR and polyPR coding sequences of indicated length $(10 x, 20 x, 30 x, 40 x, 50 x, 75 x$ or 102x) inserted into fluorescent reporter, $L-$ linker. (C) ChFP/GFP ratios for different poly-PR and poly-GR stall reporters. L- linker, pK - poly-K coded by (AAA) codons. Median GFP and ChFP fluorescence values were measured by FACS and resulted ChFP/GFP ratios were normalized to ChFP/GFP ratio of negative stall reporter (linker). Bars show means and SD, $n=3$. (D) Confocal images of HEK293T cells

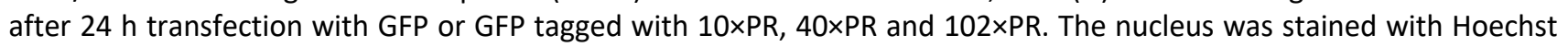
33258. Scale bar represents $10 \mu \mathrm{m}$. 

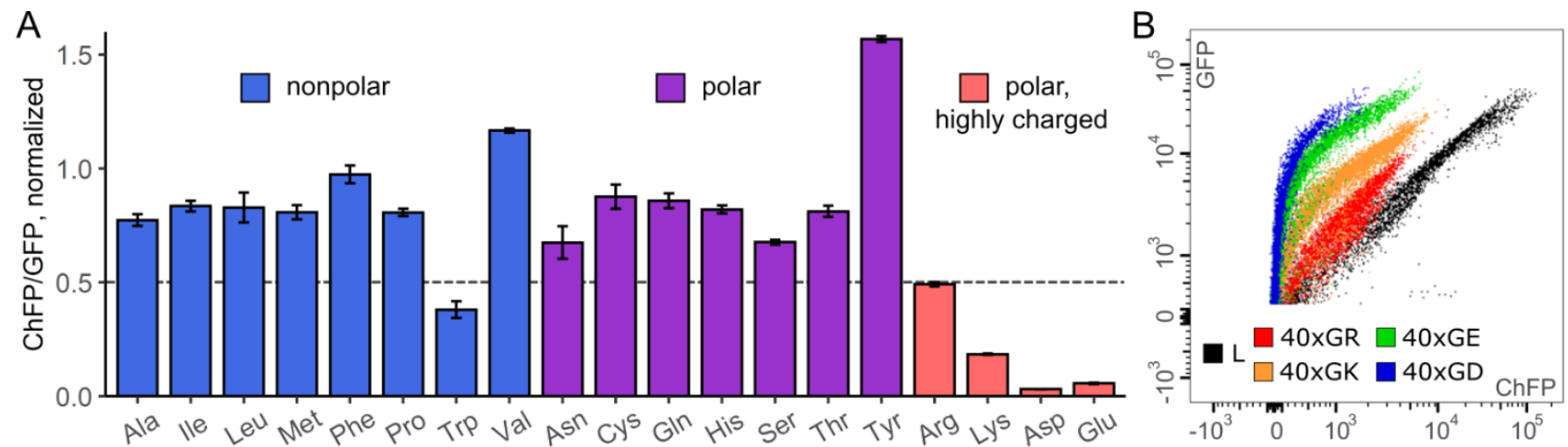

C

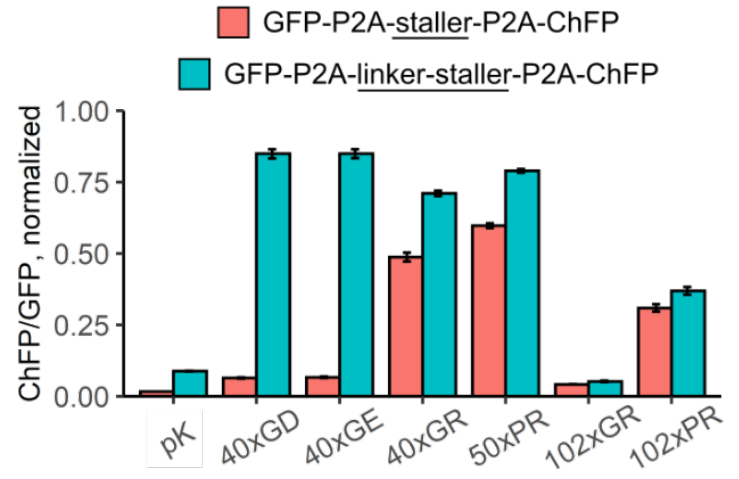

Staller in middle position VS staller at the $\mathrm{N}$-end

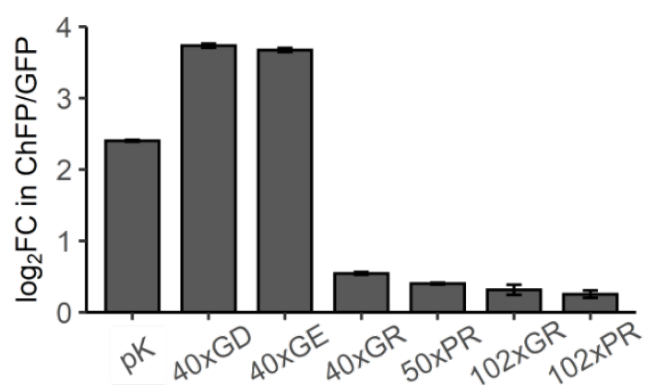

Figure 2. Ribosome stalling assays with library of different dipeptide repeats. (A) The relative ChFP/GFP ratios for different DPRs normalized to ratio in cell transfected with negative staller control (linker). Each DPR is 40xGly-Xxx, where $\mathrm{Xxx}-$ amino acid residue displayed on $\mathrm{x}$ axis. Bars show means and $\mathrm{SD}, \mathrm{n}=4$. (B) Flow chart showing decrease in ChFP readout from all fluorescent reporters containing charged DPRs: 40xGly-Arg (40xGR), 40xGly-Lys (40xGK), 40xGly-Glu

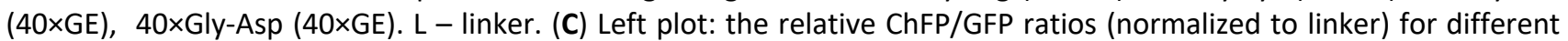
DPR stallers and poly-K (pK) that either start right after P2A sequence (pink bars) or have a linker sequence added to their $\mathrm{N}$-end (blue bars). Bars show means and SD, $\mathrm{n}=3$. Right plot: $\log 2$ fold changes in ChFP/GFP ratio after adding the linker sequence between P2A and $\mathrm{N}$-end of DPR. 
bioRxiv preprint doi: https://doi.org/10.1101/2022.02.09.479805; this version posted February 10, 2022. The copyright holder for this preprint (which was not certified by peer review) is the author/funder, who has granted bioRxiv a license to display the preprint in perpetuity. It is made available under aCC-BY-NC-ND 4.0 International license.
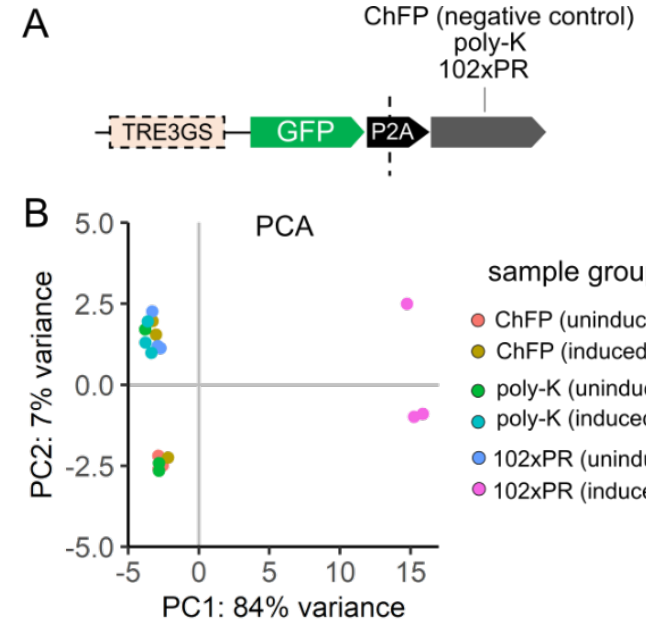

sample group

- ChFP (uninduced)

- ChFP (induced)

- poly-K (uninduced)

- poly-K (induced)

- 102xPR (uninduced)

- 102xPR (induced)

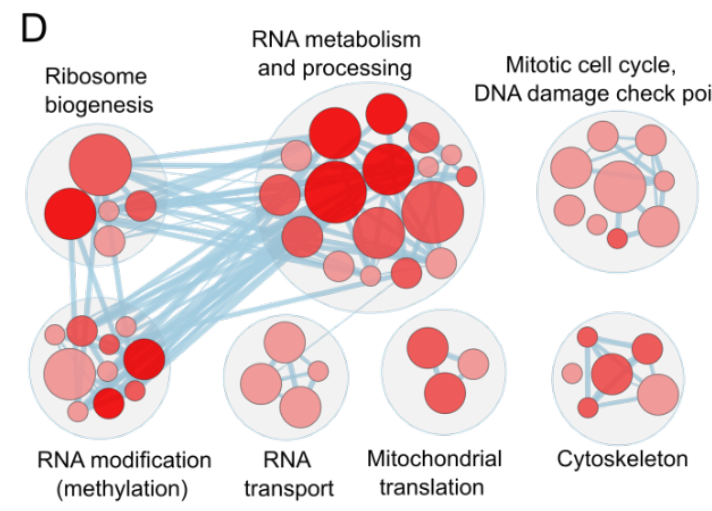

C 102xPR (induced) (compared to ChFP (induced))
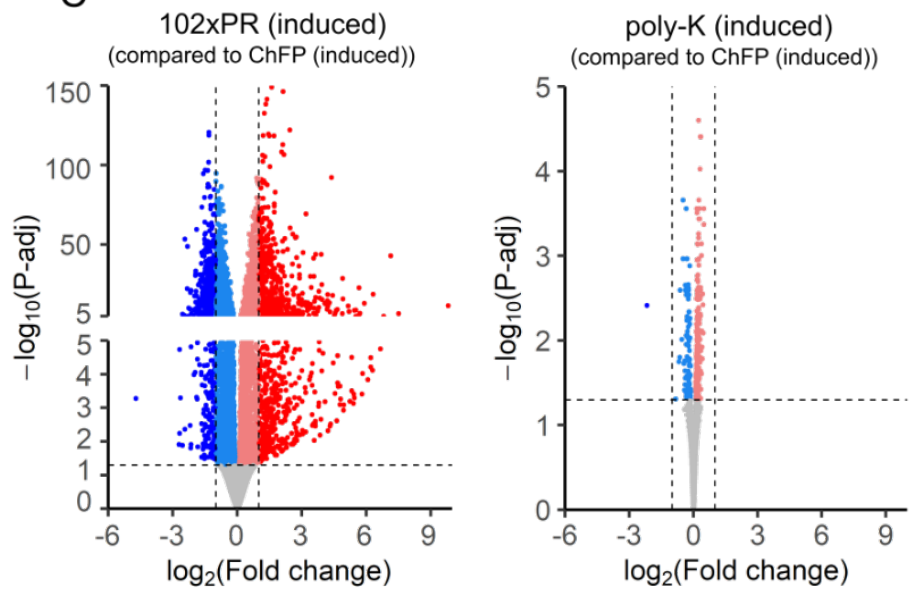

Metabolism Cellular biosynthesis, of glycoproteins other metabolic pathways

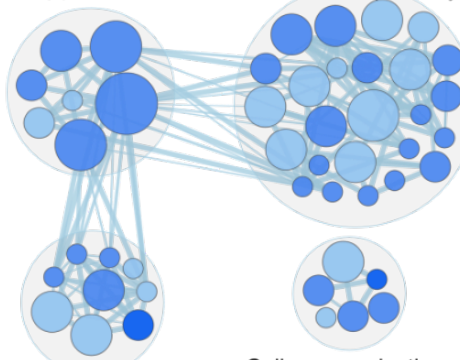

Lipid metabolism

Cell communication

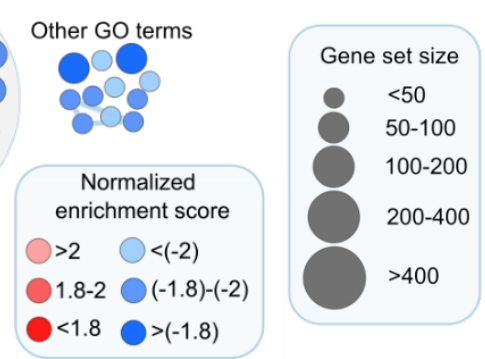

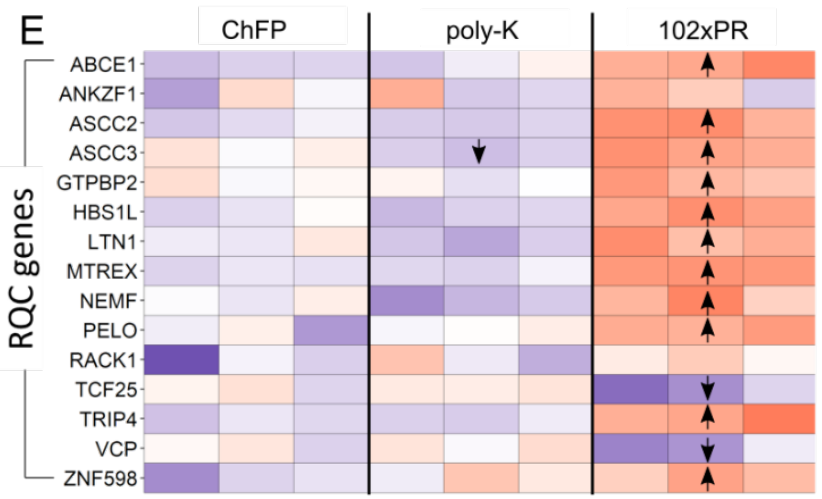

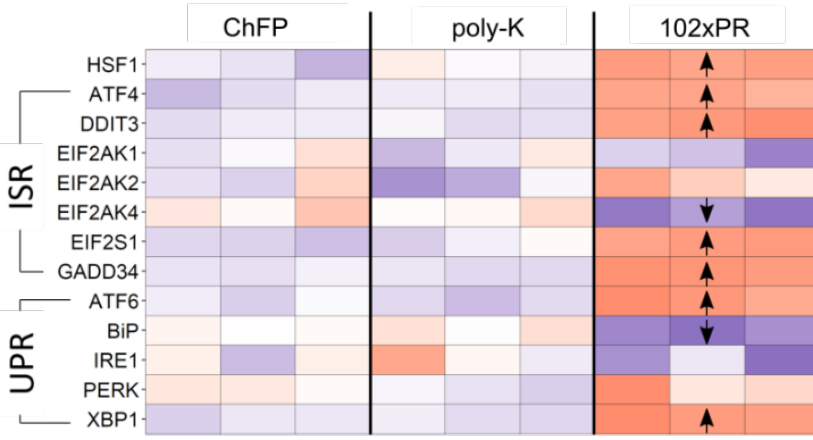

Z-score

Figure 3. Changes in global transcriptome caused by expression of poly-K or poly-PR stallers. (A) A schematic of the transcript used to generate stable cell lines. TRE3GS - promoter containing tetracycline response element (TRE). (B) Principal component analysis plot for all samples. Each group was done in triplicate $(n=3)$. (C) Volcano plots showing differentially expressed genes ( $p$-adj $<0.05$, horizontal line) for each staller. Red - upregulated genes, blue downregulated genes. Vertical lines represent cut-off values for log2(Fold change): 1 and -1. (D) Enrichment map with GO terms that were significantly up- (red) or downregulated (blue) upon 102xPR expression (FDR q-value $<0.1$ ) and grouped into the following clusters. Color intensity is proportional to the normalized enrichment score (NES) for a given GO term. Node size is proportional to a total number of genes in GO category (gene set size). (E) Heat maps with Z-scores for selected genes from various stress pathways (RQC - ribosome quality control, ISR - integrated stress response, UPR - unfolded protein response) in cells with induced expression of ChFP, poly-K coded by (AAA) codons or 102×PR. Arrows indicate differentially expressed genes (up/down arrows for up- and down-regulated genes respectively) in cells with induced staller expression when compared to ChFP-expressing cells. 


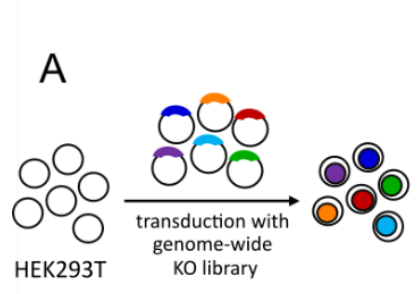

B

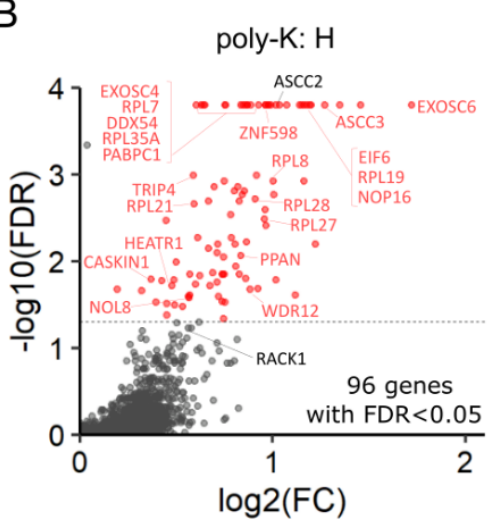

102xPR: H

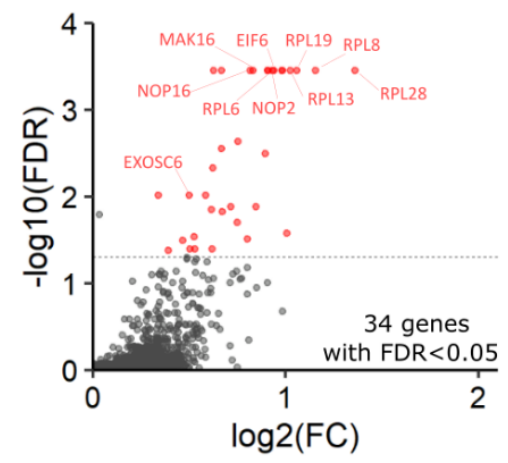

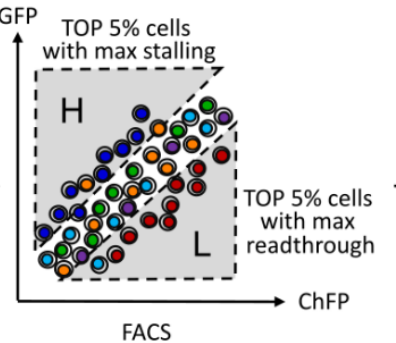

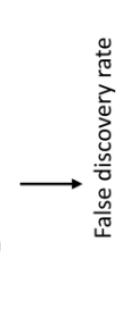

poly-K: L
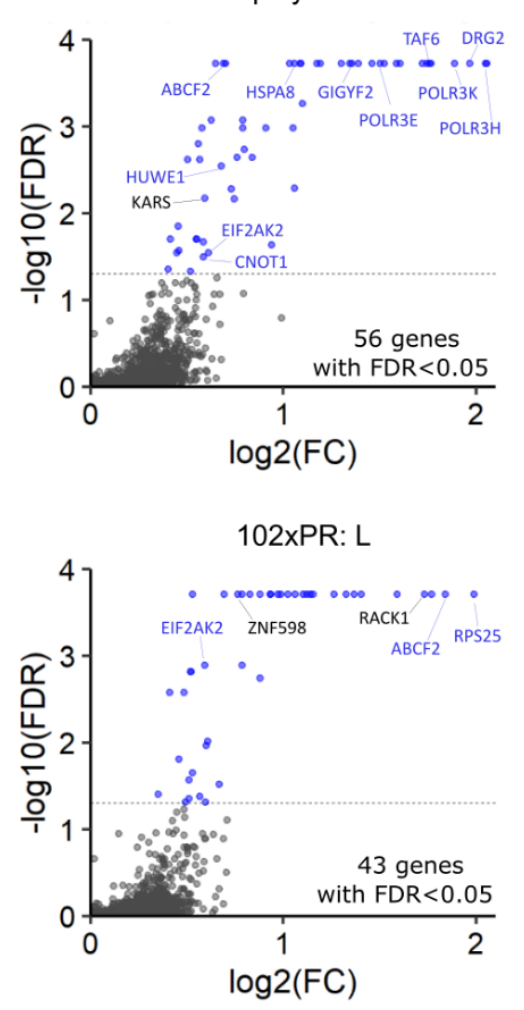

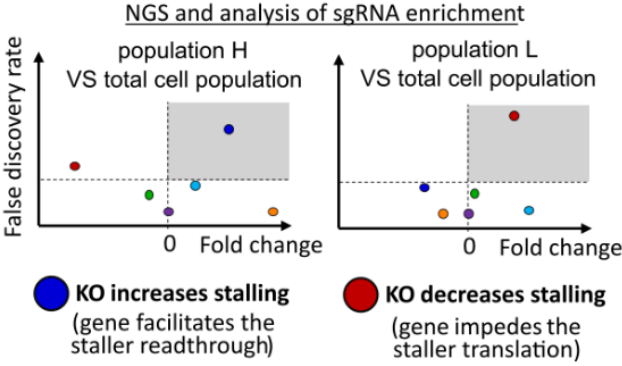

C

TOP gene KOs improving the readthrough

\begin{tabular}{|c|c|c|c|c|}
\hline \multicolumn{3}{|c|}{ poly-K } & \multirow{3}{*}{$\begin{array}{c}\text { both } \\
\text { EIF6 } \\
\text { EXOSC6 }\end{array}$} & \multirow{2}{*}{$102 \times P R$} \\
\hline ZNF598 & RPL7 & PABPC1 & & \\
\hline ASCC3 & $R P L 21$ & WDR12 & & NOP2 \\
\hline TRIP4 & RPL35A & PPAN & NOP16 & RPL6 \\
\hline DDX54 & RPL27 & NOL8 & RPL19 & RPL13 \\
\hline EXOSC4 & CASKIN1 & HEATR1 & $\begin{array}{c}R P L 28 \\
\text { RPL8 }\end{array}$ & MAK16 \\
\hline \multicolumn{5}{|c|}{ TOP gene KOs increasing stalling } \\
\hline & poly-K & & both & 102xPR \\
\hline HSPA8 & DRG2 & POLR3H & EIF2AK2 & \\
\hline GIGYF2 & CNOT1 & POLR3E & ABCF2 & RPS25 \\
\hline TAF6 & HUWE1 & POLR3K & & \\
\hline
\end{tabular}

Figure 4. Genome wide knockout screening for genes modulating stalling of poly-K and poly-PR stallers. (A) Schematic of strategy. (B) Volcano plots of gene KO enrichment. The number on the plot indicates the number of screen hits (FDR < 0.05 ) for each category. Genes which targeting sgRNAs were enriched in population $\mathrm{H}$ (highest ChFP/GFP ratio) are shown in red. Genes which targeting sgRNAs were enriched in population L (lowest ChFP/GFP ratio) are shown in blue. (C) TOP screen hits for each staller (red - KOs causing readthrough, blue - KOs increasing stalling). Genes in bold indicate genes that appeared as TOP screen hits for both stallers. 
bioRxiv preprint doi: https://doi.org/10.1101/2022.02.09.479805; this version posted February 10, 2022. The copyright holder for this preprint (which was not certified by peer review) is the author/funder, who has granted bioRxiv a license to display the preprint in perpetuity. It is made available under aCC-BY-NC-ND 4.0 International license.

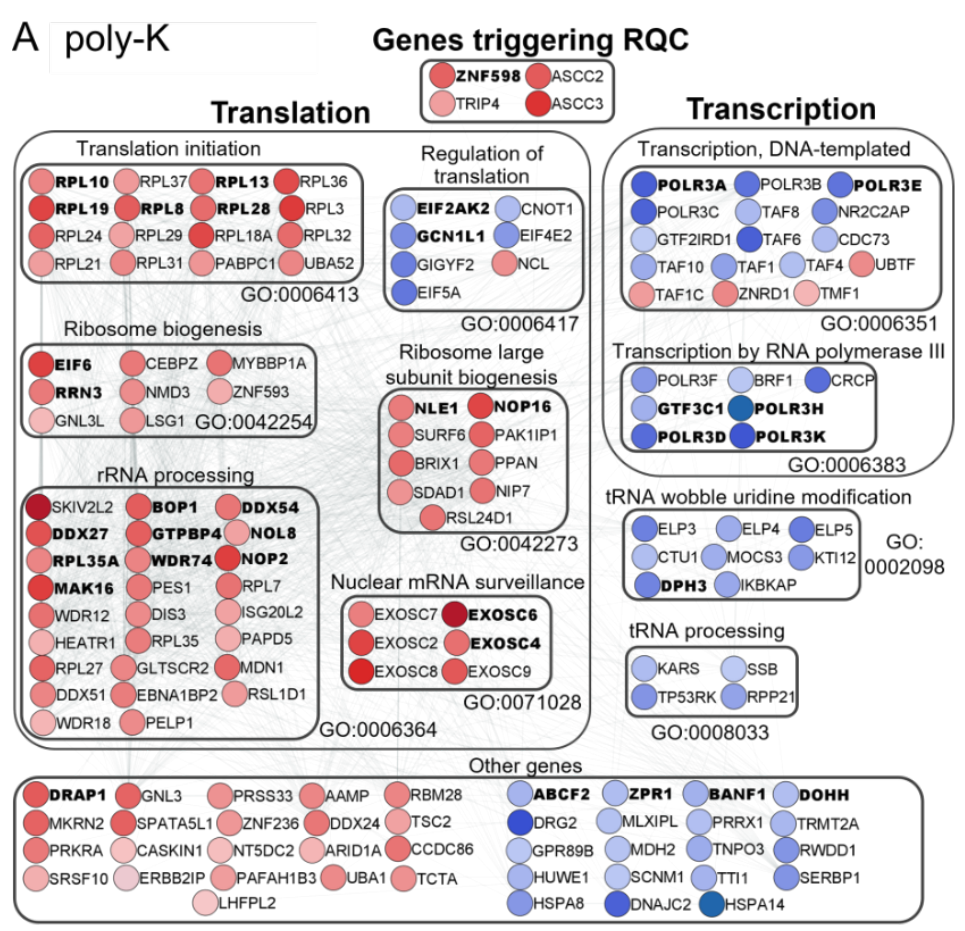

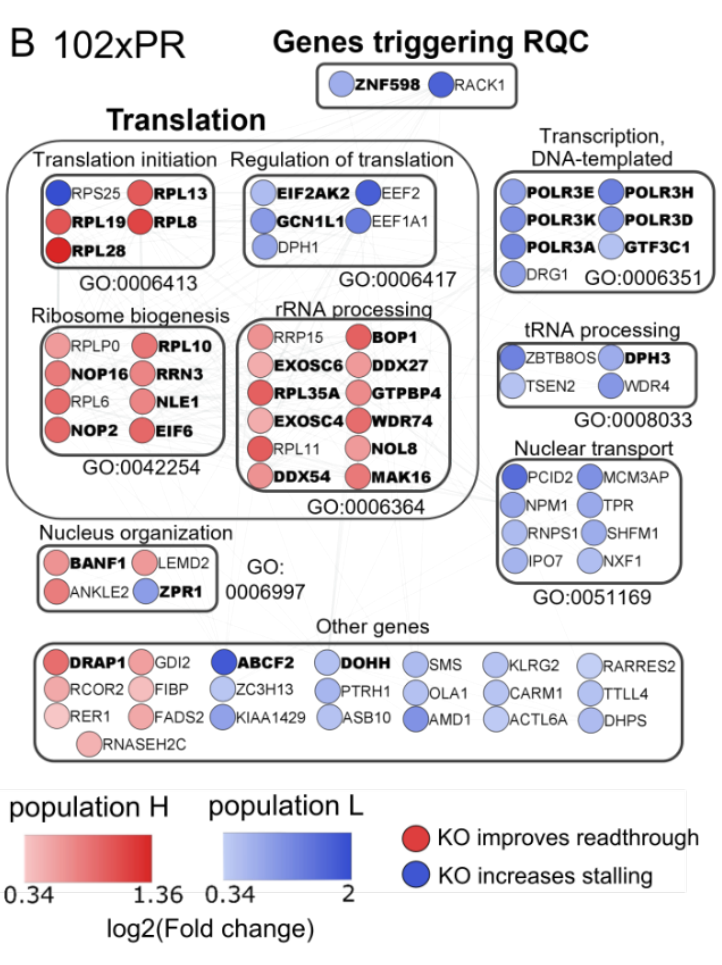

Figure 5. Top enriched gene ontology (GO) terms for screen hits at 5\% FDR. (A) poly-K, (B) 102×PR. Genes in bold were the screen hits for both stallers. 


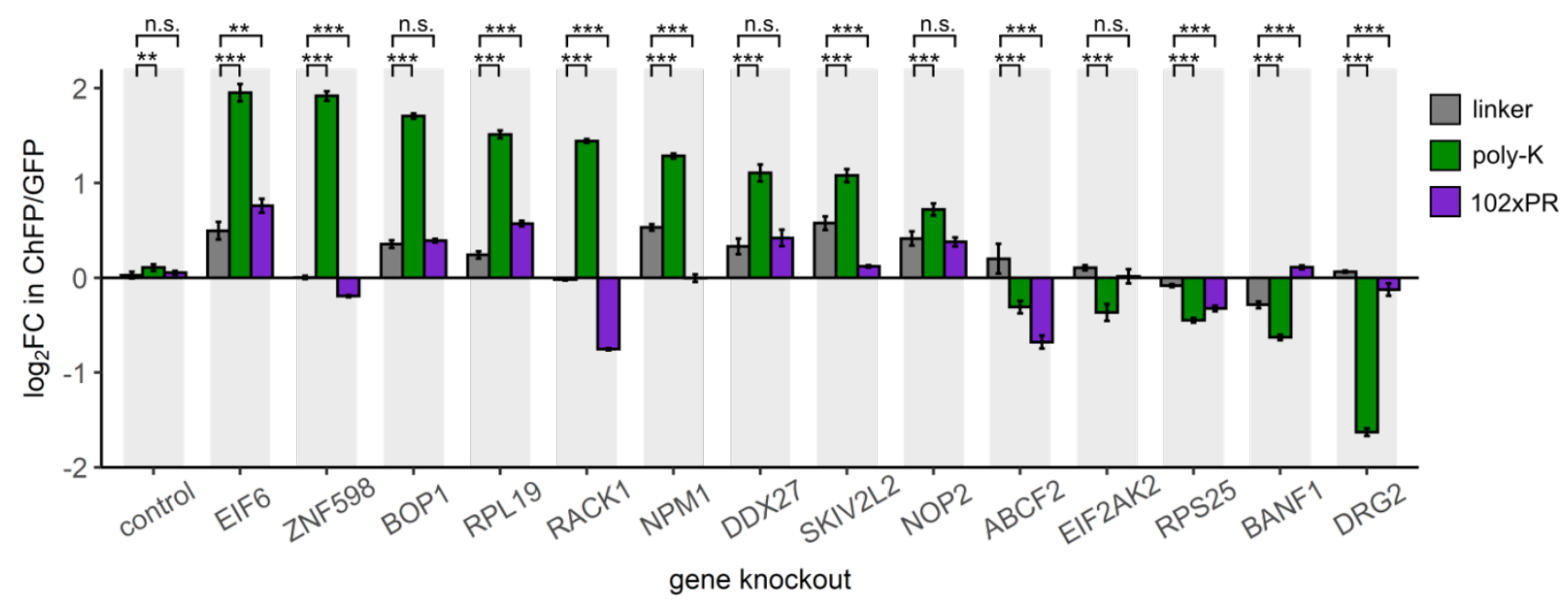

Figure 6. Log2 fold changes in ChFP/GFP ratio after KO of different genes selected from our screen hits. Control - cells expressing non-targeting sgRNA. Bars show means and SD, $n=4$. For each gene knockout, log2 fold changes for both stallers were compared to log2 fold changes for a linker construct using one-way ANOVA and Dunnett's post hoc test. n.s. - not significant; ${ }^{* *} \mathrm{P}<0.01 ;{ }^{* * *} \mathrm{P}<0.0001$. 
A

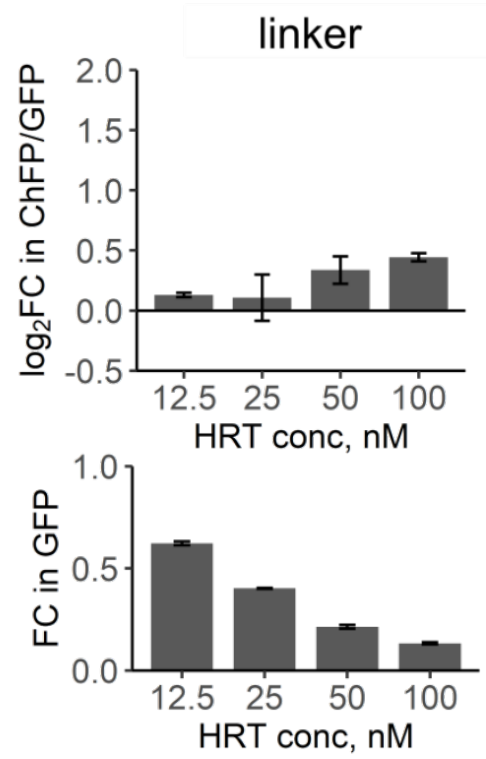

B
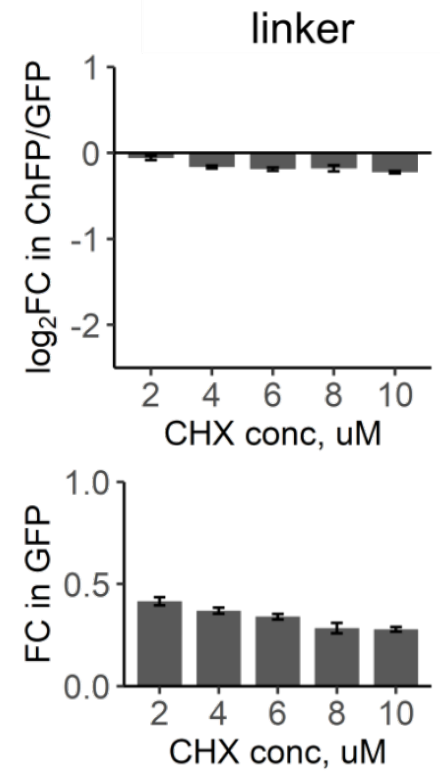
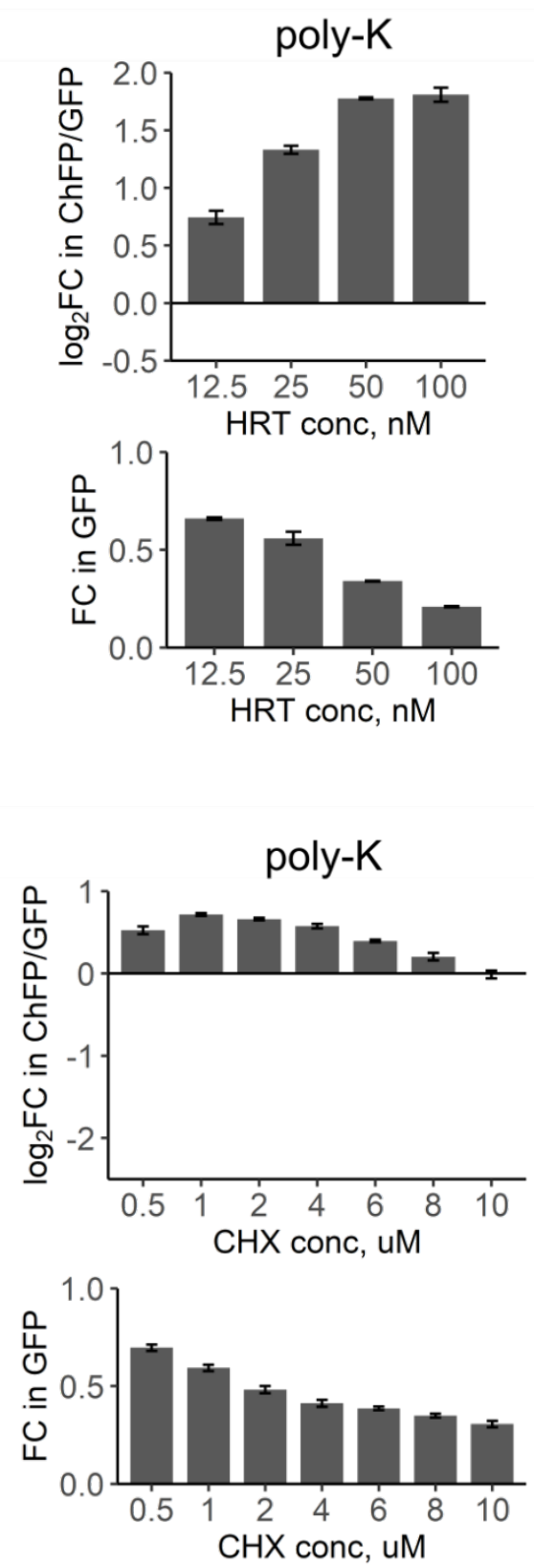
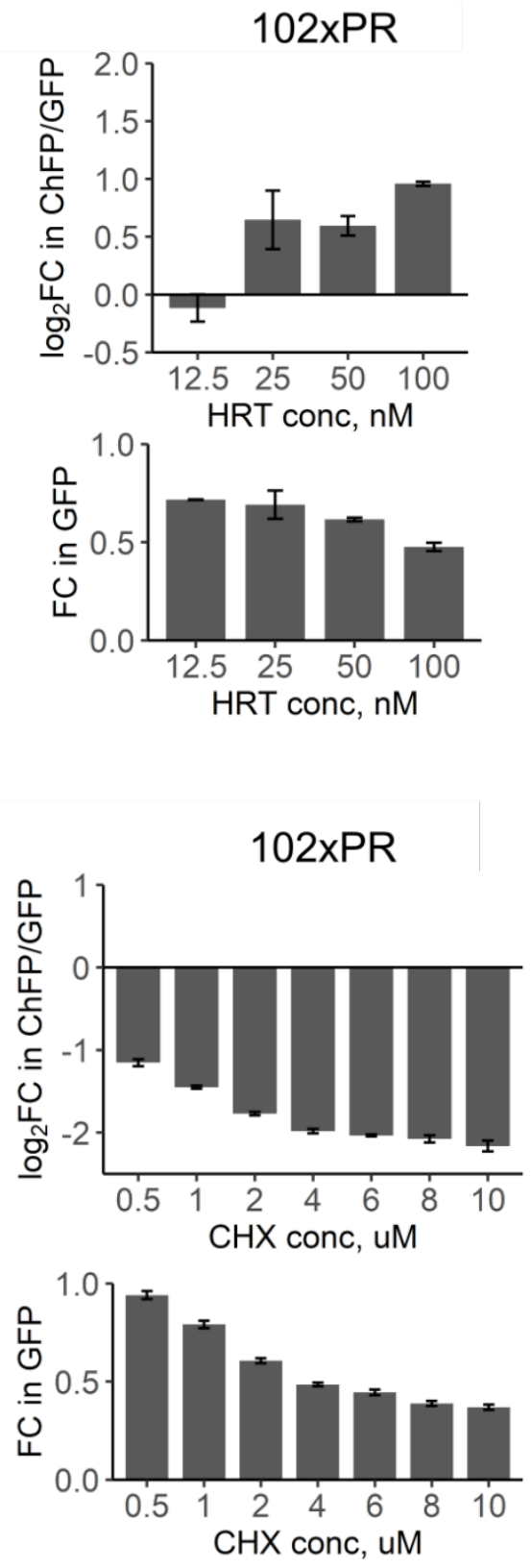

Figure 7. The effect of translation inhibition on the readthrough of different stallers. HEK293T cells were transfected with different fluorescent stall reporters and various concentrations of translation inhibitor were added $6 \mathrm{~h}$ after transfection before visible formation of fluorescent proteins. Cells were subjected to FACS after $18 \mathrm{~h}$ of treatment. Top plots on each panel show log2 fold change in ChFP/GFP ratio in cells treated with indicated concentration of translation inhibitor compared to untreated cells. Bottom plots on each panel show fold change in GFP fluorescence compared to untreated cells. Bars show means and SD, $n=3$. (A) Harringtonine, inhibitor of translation initiation. (B) Cycloheximide, inhibitor of translation elongation. 